\title{
Radiative decay of hadronic molecule state for quarks
}

\author{
Xiaozhao Chen $\odot,{ }^{1, *}$ Xiaofu Lü,${ }^{2,3,4}$ Renbin Shi, ${ }^{1}$ Xiurong Guo, ${ }^{1}$ and Qingbiao Wang ${ }^{1}$ \\ ${ }^{1}$ Department of Foundational Courses, Shandong University of Science and Technology, \\ Taian, 271019, China \\ ${ }^{2}$ Department of Physics, Sichuan University, Chengdu, 610064, China \\ ${ }^{3}$ Institute of Theoretical Physics, The Chinese Academy of Sciences, Beijing 100080, China \\ ${ }^{4}$ CCAST (World Laboratory), P.O. Box 8730, Beijing 100080, China
}

(Received 24 October 2018; revised manuscript received 11 November 2019; published 14 January 2020)

\begin{abstract}
Using the general form of the generalized Bethe-Salpeter wave functions for four-quark states describing the meson-meson molecular structure given in our previous work, we obtain the general formulas for the decay widths of molecular states composed of two vector mesons with arbitrary spin and parity into two photons. Then, this general formalism is applied to investigate the radiative two-photon decay of the observed $X(3915)$ state, in which this exotic state $X(3915)$ is considered as a mixed state of two components $D^{* 0} \bar{D}^{* 0}$ and $D^{*+} D^{*-}$. The numerical result of decay mode $X(3915) \rightarrow \gamma \gamma$ is consistent with the experimental values.
\end{abstract}

DOI: 10.1103/PhysRevD.101.014009

\section{INTRODUCTION}

In the framework of $\mathrm{QCD}$, beyond the quark-antiquark $(q \bar{q})$ state, there should be other internal structures, such as the tetraquark state, molecular state, etc., which have been used to interpret exotic mesons [1-6]. It is most reasonable and fascinating to study the structure of exotic meson from QCD. In our previous works [7-9], we have carefully investigated the molecular state composed of two vector mesons as far as possible from QCD and obtained the general form of generalized Bethe-Salpeter (GBS) wave functions of molecular states as four-quark states. This GBS wave function for the four-quark state was applied to evaluate the strong decay width of a molecular state composed of two heavy vector mesons into a heavy meson plus a light meson in Ref. [9], while the radiative decay of a molecular state has still not been investigated. In this paper, we will emphatically investigate the two-photon decay of a molecular state composed of two vector mesons.

Different from the previous works [1-3] about hadronic molecule states, in our approach, the vector mesons in a molecular state are considered as bound states composed of a quark and an antiquark. Because of the spontaneous breaking of chiral symmetry, the effective interaction

\footnotetext{
* Corresponding author. chen_xzhao@sina.com

Published by the American Physical Society under the terms of the Creative Commons Attribution 4.0 International license. Further distribution of this work must maintain attribution to the author(s) and the published article's title, journal citation, and DOI. Funded by SCOAP ${ }^{3}$.
}

Lagrangian at low-energy QCD can be regarded as a Lagrangian for the interaction of light mesons with quarks. According to the effective theory at low-energy QCD, we can investigate the exchanged meson interaction with quarks in the vector meson and obtain the interaction kernel between two vector mesons. Solving the BetheSalpeter (BS) equation, we obtained the masses and BS wave functions of molecular states composed of two vector mesons $[7,8]$. From the molecule state model, we gave the GBS wave function for the four-quark state [9]. The GBS wave function derived from QCD is an essential prerequisite to accurately calculating the decay widths of a molecular state containing strong and radiative decays.

When investigating the radiative decay of a molecular state composed of two vector mesons, we still consider the internal structure of these vector mesons. The decay interaction is derived from the photon interaction with quarks in these vector mesons. The photon-quark interaction can be described by the exact interaction Lagrangian $\mathcal{L}_{I}=i \frac{n}{3} e \bar{q} \gamma_{\mu} q A_{\mu}$, where the value of $n$ is determined by the flavor of the quark field. In this work, we investigate the radiative two-photon decay of the molecular state. The lowest-order approximation for this decay mode is the second-order S-matrix element. Considering this radiative decay interaction and using the GBS wave function, we can obtain the general formulas for the matrix elements of the charge current between the fourquark state and vacuum. Finally, the two-photon decay width of the molecular state can be evaluated.

Then, this approach is used to investigate a significant process: the radiative two-photon decay of $X(3915)[10,11]$. The experimental data of $X(3915)$, once named $Y(3940)$ $[12,13]$, introduces a new challenge to the ordinary $c \bar{c}$ 
charmonium interpretation $[14,15]$. Moreover, the spinparity quantum numbers $J^{P}$ of $X(3915)$ are not unambiguously determined in experiment [16]. In this paper, we assume that this exotic meson $X(3915)$ is a mixed state of two components $D^{* 0} \bar{D}^{* 0}$ and $D^{*+} D^{*-}$ with $J^{P}=0^{+}$or $2^{+}$. From the effective theory at low-energy QCD and the Weinberg-Salam model, we comprehensively and systematically analyze the radiative two-photon decay of a mixed state consisting of two components $D^{* 0} \bar{D}^{* 0}$ and $D^{*+} D^{*-}$. The matrix elements between the four-quark state and vacuum and the radiative decay width can be calculated without an extra parameter. The calculated decay width $\Gamma(X(3915) \rightarrow \gamma \gamma)$ for $2^{+}$is more consistent with the experimental values. Therefore, our approach provides further verification for the effective theory at low-energy QCD and proves the reasonableness of the molecular hypothesis for $X(3915)$. Comparing our numerical results with experimental data, we can further discriminate the quantum numbers $J^{P}$ of the $X(3915)$ state. The BetheSalpeter theory is a relativistic theory for a two-body bound state in quantum field theory, and our approach is an generalization of BS theory. So, our approach is in fact a nonperturbative method, which can be applied to investigate arbitrary meson-meson molecular structure.

The structure of this article is as follows. In Sec. II, the GBS wave function of a molecular state as a four-quark state is given. Section III gives the general formulas for the matrix elements of the charge current between the fourquark state and vacuum. In Sec. IV, our approach is used to investigate the decay mode $X(3915) \rightarrow \gamma \gamma$. Our numerical results are presented in Sec. V, and we make some concluding remarks in Sec. VI.

\section{GBS WAVE FUNCTION FOR FOUR-QUARK STATE}

If a bound state with spin $j$ and parity $\eta_{P}$ is composed of four quarks, its GBS wave function can be defined as [9]

$\chi_{P}^{j}\left(x_{1}, x_{3}, x_{4}, x_{2}\right)=\left\langle 0\left|T \mathcal{Q}^{C}\left(x_{1}\right) \overline{\mathcal{Q}}^{A}\left(x_{3}\right) \mathcal{Q}^{B}\left(x_{4}\right) \overline{\mathcal{Q}}^{D}\left(x_{2}\right)\right| P\right\rangle$,

where $P$ is the momentum of the four-quark bound state, $\mathcal{Q}$ is the quark operator, and its superscript is a flavor label. From translational invariance, this GBS wave function can be written as

$\chi_{P}^{j}\left(x_{1}, x_{3}, x_{4}, x_{2}\right)=\frac{1}{(2 \pi)^{3 / 2}} \frac{1}{\sqrt{2 E(P)}} e^{i P \cdot X} \chi_{P}^{j}\left(X^{\prime}, x, x^{\prime}\right)$,

where $\quad E(p)=\sqrt{\mathbf{p}^{2}+m^{2}}, \quad X=\eta_{1}\left(\eta_{1}^{\prime \prime} x_{1}+\eta_{3}^{\prime \prime} x_{3}\right)+$ $\eta_{2}\left(\eta_{4}^{\prime \prime} x_{4}+\eta_{2}^{\prime \prime} x_{2}\right), \quad X^{\prime}=\left(\eta_{1}^{\prime \prime} x_{1}+\eta_{3}^{\prime \prime} x_{3}\right)-\left(\eta_{4}^{\prime \prime} x_{4}+\eta_{2}^{\prime \prime} x_{2}\right)$, $x=x_{1}-x_{3}, x^{\prime}=x_{2}-x_{4}, \eta_{1}+\eta_{2}=1, \eta_{1,3}^{\prime \prime}=m_{C, A} /\left(m_{C}+m_{A}\right)$, $\eta_{2,4}^{\prime \prime}=m_{D, B} /\left(m_{D}+m_{B}\right)$ and $m_{A, B, C, D}$ are the quark masses. Then, making the Fourier transformation, we obtain the GBS wave function of the four-quark bound state in the momentum representation

$$
\begin{aligned}
\chi_{P}^{j}( & \left.p_{1}, p_{3}, p_{4}, p_{2}\right) \\
= & \int d^{4} x_{1} d^{4} x_{3} d^{4} x_{4} d^{4} x_{2} \chi_{P}^{j}\left(x_{1}, x_{3}, x_{4}, x_{2}\right) \\
& \times e^{-i p_{1} \cdot x_{1}} e^{-i p_{3} \cdot x_{3}} e^{-i p_{4} \cdot x_{4}} e^{-i p_{2} \cdot x_{2}} \\
= & \frac{1}{(2 \pi)^{3 / 2}} \frac{1}{\sqrt{2 E(P)}}(2 \pi)^{4} \delta^{(4)}\left(P-p_{1}-p_{3}-p_{4}-p_{2}\right) \\
& \times \chi^{j}\left(P, p, k, k^{\prime}\right),
\end{aligned}
$$

where $p_{1}, p_{3}, p_{4}$, and $p_{2}$ are the momenta carried by the fields $\mathcal{Q}^{C}, \overline{\mathcal{Q}}^{A}, \mathcal{Q}^{B}$, and $\overline{\mathcal{Q}}^{D} ; p, k$, and $k^{\prime}$ are the conjugate variables to $X^{\prime}, x$, and $x^{\prime}$, respectively; and $p=$ $\eta_{2}\left(p_{1}+p_{3}\right)-\eta_{1}\left(p_{4}+p_{2}\right), \quad k=\eta_{3}^{\prime \prime} p_{1}-\eta_{1}^{\prime \prime} p_{3}$, and $k^{\prime}=$ $\eta_{4}^{\prime \prime} p_{2}-\eta_{2}^{\prime \prime} p_{4}$. In the hadronic molecule structure, $p$ is the relative momentum between two mesons in the molecular state, and $k$ and $k^{\prime}$ are the relative momenta between the quark and antiquark in these two mesons, respectively, shown in Fig. 1. This work aims to investigate the molecular state composed of two vector mesons. In Fig. 1, $V$ represents the vector meson with mass $M_{1}, \bar{V}^{\prime}$ represents the antiparticle of the vector meson $V^{\prime}$ with mass $M_{2}$, and $M S$ represents the vector-vector molecular state.

In Fig. 1, there are three two-body systems in molecular state: a meson-meson bound state and two quark-antiquark bound states. We define the BS wave functions of these two-body systems as $\chi_{P}^{j}\left(p_{1}^{\prime}, p_{2}^{\prime}\right), \quad \chi_{p_{1}^{\prime}}\left(p_{1}, p_{3}\right)$, and $\chi_{p_{2}^{\prime}}\left(p_{4}, p_{2}\right)$, respectively. According to the $\mathrm{BS}$ theory, the BS wave function for the bound state of two vector mesons has the form

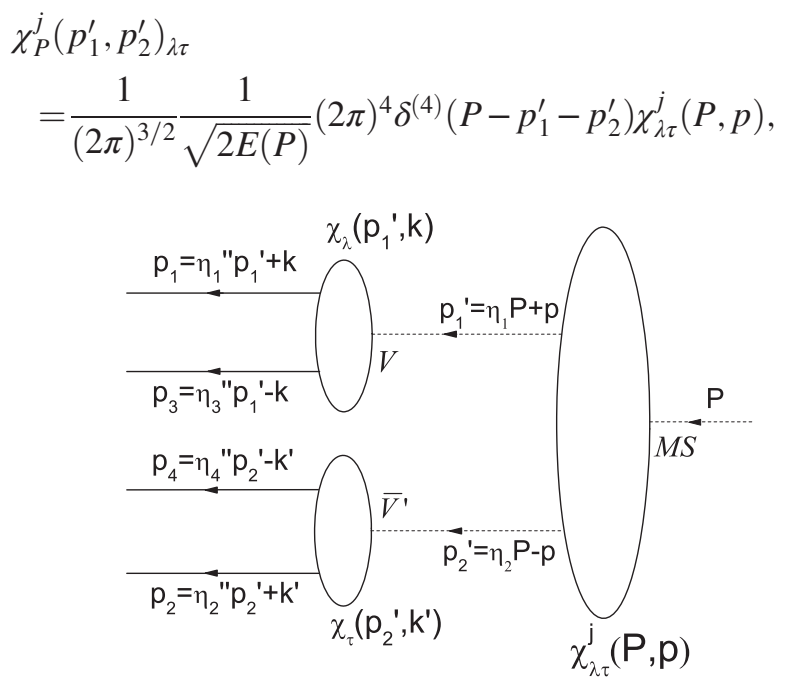

FIG. 1. Generalized Bethe-Salpeter wave function for the fourquark state in the momentum representation. The solid lines denote quark propagators, and the unfilled ellipses represent Bethe-Salpeter amplitudes. 
and the BS wave functions of two vector mesons are

$$
\begin{aligned}
\chi_{p_{1}^{\prime}}\left(p_{1}, p_{3}\right)_{\lambda}= & \frac{1}{(2 \pi)^{3 / 2}} \frac{1}{\sqrt{2 E\left(p_{1}^{\prime}\right)}}(2 \pi)^{4} \\
& \times \delta^{(4)}\left(p_{1}^{\prime}-p_{1}-p_{3}\right) \chi_{\lambda}\left(p_{1}^{\prime}, k\right), \\
\chi_{p_{2}^{\prime}}\left(p_{4}, p_{2}\right)_{\tau}= & \frac{1}{(2 \pi)^{3 / 2}} \frac{1}{\sqrt{2 E\left(p_{2}^{\prime}\right)}}(2 \pi)^{4} \\
& \times \delta^{(4)}\left(p_{2}^{\prime}-p_{4}-p_{2}\right) \chi_{\tau}\left(p_{2}^{\prime}, k^{\prime}\right),
\end{aligned}
$$

where $p_{1}^{\prime}$ and $p_{2}^{\prime}$ are the momenta of two vector mesons, and we have $p_{1}^{\prime}=\eta_{1} P+p, p_{2}^{\prime}=\eta_{2} P-p$ and $\eta_{1,2}=$ $M_{1,2} /\left(M_{1}+M_{2}\right)$. Applying the Feynman rules and comparing with Eq. (3), we obtain the GBS wave function for the four-quark state describing the molecular state composed of two vector mesons with arbitrary spin and definite parity [9],
$\chi^{j}\left(P, p, k, k^{\prime}\right)=(2 \pi)^{8} \chi_{\lambda}\left(p_{1}^{\prime}, k\right) \chi_{\lambda \tau}^{j}(P, p) \chi_{\tau}\left(p_{2}^{\prime}, k^{\prime}\right)$.

In Ref. [7], we considered a bound state composed of two massive vector fields with spin $j$ and parity $\eta_{P}$ and defined its BS wave function in the momentum representation $\chi_{\lambda \tau}^{j}(P, p)$. The polarization tensor of the bound state $\eta_{\mu_{1} \mu_{2} \cdots \mu_{j}}$ can be separated,

$$
\chi_{\lambda \tau}^{j}(P, p)=\eta_{\mu_{1} \mu_{2} \cdots \mu_{j}} \chi_{\mu_{1} \mu_{2} \cdots \mu_{j} \lambda \tau}(P, p),
$$

where the subscripts $\lambda$ and $\tau$ are derived from these two vector fields. The polarization tensor $\eta_{\mu_{1} \mu_{2} \cdots \mu_{j}}$ describes the spin of the bound state, which is totally symmetric, transverse, and traceless:

$\eta_{\mu_{1} \mu_{2} \ldots}=\eta_{\mu_{2} \mu_{1} \ldots,}, \quad P_{\mu_{1}} \eta_{\mu_{1} \mu_{2} \ldots}=0, \quad \eta_{\mu_{1} \mu_{2} \cdots}^{\mu_{1}}=0$.

From Lorentz covariance, we have

$$
\begin{aligned}
\chi_{\mu_{1} \cdots \mu_{j} \lambda \tau}= & p_{\mu_{1}} \cdots p_{\mu_{j}}\left[g_{\lambda \tau} f_{1}+\left(P_{\lambda} p_{\tau}+P_{\tau} p_{\lambda}\right) f_{2}+\left(P_{\lambda} p_{\tau}-P_{\tau} p_{\lambda}\right) f_{3}+P_{\lambda} P_{\tau} f_{4}+p_{\lambda} p_{\tau} f_{5}\right] \\
& +\left(p_{\left\{\mu_{2}\right.} \cdots p_{\mu_{j}} g_{\left.\mu_{1}\right\} \lambda} p_{\tau}+p_{\left\{\mu_{2}\right.} \cdots p_{\mu_{j}} g_{\left.\mu_{1}\right\} \tau} p_{\lambda}\right) f_{6}+\left(p_{\left\{\mu_{2}\right.} \cdots p_{\mu_{j}} g_{\left.\mu_{1}\right\} \lambda} p_{\tau}-p_{\left\{\mu_{2}\right.} \cdots p_{\mu_{j}} g_{\left.\mu_{1}\right\} \tau} p_{\lambda}\right) f_{7} \\
& +\left(p_{\left\{\mu_{2}\right.} \cdots p_{\mu_{j}} g_{\left.\mu_{1}\right\} \lambda} P_{\tau}+p_{\left\{\mu_{2}\right.} \cdots p_{\mu_{j}} g_{\left.\mu_{1}\right\} \tau} P_{\lambda}\right) f_{8}+\left(p_{\left\{\mu_{2}\right.} \cdots p_{\mu_{j}} g_{\left.\mu_{1}\right\} \lambda} P_{\tau}-p_{\left\{\mu_{2}\right.} \cdots p_{\mu_{j}} g_{\left.\mu_{1}\right\} \tau} P_{\lambda}\right) f_{9} \\
& +p_{\mu_{1}} \cdots p_{\mu_{j}} \epsilon_{\lambda \tau \xi \zeta} p_{\xi} P_{\zeta} f_{10}+p_{\left\{\mu_{2}\right.} \cdots p_{\mu_{j}} \epsilon_{\left.\mu_{1}\right\} \lambda \tau \xi} p_{\xi} f_{11}+p_{\left\{\mu_{2}\right.} \cdots p_{\mu_{j}} \epsilon_{\left.\mu_{1}\right\} \lambda \tau \xi} P_{\xi} f_{12} \\
& +\left(p_{\left\{\mu_{2}\right.} \cdots p_{\mu_{j}} \epsilon_{\left.\mu_{1}\right\} \lambda \xi \zeta} p_{\xi} P_{\zeta} p_{\tau}+p_{\left\{\mu_{2}\right.} \cdots p_{\mu_{j}} \epsilon_{\left.\mu_{1}\right\} \tau \xi \zeta} p_{\xi} P_{\zeta} p_{\lambda}\right) f_{13} \\
& +\left(p_{\left\{\mu_{2}\right.} \cdots p_{\mu_{j}} \epsilon_{\left.\mu_{1}\right\} \lambda \xi \zeta} p_{\xi} P_{\zeta} p_{\tau}-p_{\left\{\mu_{2}\right.} \cdots p_{\mu_{j}} \epsilon_{\left.\mu_{1}\right\} \tau \xi \zeta} p_{\xi} P_{\zeta} p_{\lambda}\right) f_{14} \\
& +\left(p_{\left\{\mu_{2}\right.} \cdots p_{\mu_{j}} \epsilon_{\left.\mu_{1}\right\} \lambda \xi \zeta} p_{\xi} P_{\zeta} P_{\tau}+p_{\left\{\mu_{2}\right.} \cdots p_{\mu_{j}} \epsilon_{\left.\mu_{1}\right\} \tau \xi \zeta} p_{\xi} P_{\zeta} P_{\lambda}\right) f_{15} \\
& +\left(p_{\left\{\mu_{2}\right.} \cdots p_{\mu_{j}} \epsilon_{\left.\mu_{1}\right\} \lambda \xi \zeta} p_{\xi} P_{\zeta} P_{\tau}-p_{\left\{\mu_{2}\right.} \cdots p_{\mu_{j}} \epsilon_{\left.\mu_{1}\right\} \tau \xi \zeta} p_{\xi} P_{\zeta} P_{\lambda}\right) f_{16} \\
& +p_{\mu_{3}} \cdots p_{\mu_{j}} g_{\mu_{1} \lambda} g_{\mu_{2} \tau} f_{17},
\end{aligned}
$$

where $\left\{\mu_{1}, \ldots, \mu_{j}\right\}$ represents symmetrization of the indices $\mu_{1}, \ldots, \mu_{j}$. In fact, the relative momenta $p_{\mu_{1}}, \ldots, p_{\mu_{j}}, p_{\lambda}, p_{\tau}$ represent the orbital angular momenta. There should be 17 scalar functions $f_{i}\left(P \cdot p, p^{2}\right)(i=1, \ldots, 17)$ in Eq. (10). In Eq. (6) of Ref. [7], a tensor structure is omitted. In this paper, the missing term is added as the last term in Eq. (10). Using the subsidiary condition for the massive vector field $\partial_{\mu} A_{\mu}(x)=0$ and the equal-time commutation relation, we can obtain

$$
p_{1 \lambda}^{\prime} \chi_{\lambda \tau}^{j}(P, p)=p_{2 \tau}^{\prime} \chi_{\lambda \tau}^{j}(P, p)=0
$$

The proof has been given by Refs. [7,17]. Then, considering the properties of the BS wave function under space reflection, we obtain the revised general form of the BS wave functions for the bound states composed of two massive vector fields with arbitrary spin and definite parity (see details in Ref. [7]), for $\eta_{P}=(-1)^{j}$,

$$
\begin{aligned}
\chi_{\lambda \tau}^{j=0}(P, p)= & \frac{1}{\mathcal{N}^{j}}\left(T_{\lambda \tau}^{1} \phi_{1}+T_{\lambda \tau}^{2} \phi_{2}\right), \\
\chi_{\lambda \tau}^{j \neq 0}(P, p)= & \frac{1}{\mathcal{N}^{j}} \eta_{\mu_{1} \cdots \mu_{j}}\left[p_{\mu_{1}} \cdots p_{\mu_{j}}\left(T_{\lambda \tau}^{1} \phi_{1}+T_{\lambda \tau}^{2} \phi_{2}\right)\right. \\
& \left.+T_{\mu_{1} \cdots \mu_{j} \lambda \tau}^{3} \phi_{3}+T_{\mu_{1} \cdots \mu_{j} \lambda \tau}^{4} \phi_{4}+T_{\mu_{1} \cdots \mu_{j} \lambda \tau}^{5} \phi_{5}\right],
\end{aligned}
$$

and for $\eta_{P}=(-1)^{j+1}$,

$$
\chi_{\lambda \tau}^{j=0}(P, p)=\frac{1}{\mathcal{N}^{j}} \epsilon_{\lambda \tau \xi \zeta} p_{\xi} P_{\zeta} \psi_{1},
$$

$$
\begin{aligned}
\chi_{\lambda \tau}^{j \neq 0}(P, p)= & \frac{1}{\mathcal{N}^{j}} \eta_{\mu_{1} \cdots \mu_{j}}\left(p_{\mu_{1}} \cdots p_{\mu_{j}} \epsilon_{\lambda \tau \xi \zeta} p_{\xi} P_{\zeta} \psi_{1}+T_{\mu_{1} \cdots \mu_{j} \lambda \tau}^{6} \psi_{2}\right. \\
& \left.+T_{\mu_{1} \cdots \mu_{j} \lambda \tau}^{7} \psi_{3}+T_{\mu_{1} \cdots \mu_{j} \lambda \tau}^{8} \psi_{4}+T_{\mu_{1} \cdots \mu_{j} \lambda \tau}^{9} \psi_{5}\right),
\end{aligned}
$$

where $\mathcal{N}^{j}$ is the normalization, the independent tensor structures $T_{\lambda \tau}^{i}$ are given in the Appendix A, and $\phi_{i}\left(P \cdot p, p^{2}\right)$ 
and $\psi_{i}\left(P \cdot p, p^{2}\right)$ are independent scalar functions. The scalar functions $f_{i}$ in Eq. (10) are the linear combinations of $\phi_{i}$ and $\psi_{i}$. In Eq. (18) of Ref. [7], a tensor structure is also omitted, and this error has been revised as Eq. (13) in this paper. For the vector mesons, the authors of Refs. [18-21] have obtained their BS amplitudes in Euclidean space [20,21],

$$
\begin{aligned}
\Gamma_{\lambda}^{V}\left(p_{1}^{\prime} ; k\right) & =\frac{1}{\mathcal{N}^{V}}\left(\gamma_{\lambda}+p_{1 \lambda}^{\prime} \frac{\gamma \cdot p_{1}^{\prime}}{M_{V}^{2}}\right) \varphi_{V}\left(k^{2}\right), \\
\Gamma_{\tau}^{\bar{V}^{\prime}}\left(p_{2}^{\prime} ; k^{\prime}\right) & =\frac{1}{\mathcal{N}^{\bar{V}^{\prime}}}\left(\gamma_{\tau}+p_{2 \tau}^{\prime} \frac{\gamma \cdot p_{2}^{\prime}}{M_{\bar{V}^{\prime}}^{2}}\right) \varphi_{\bar{V}^{\prime}}\left(k^{\prime 2}\right),
\end{aligned}
$$

where $\Gamma_{\lambda}^{V}\left(p_{1}^{\prime} ; k\right)$ and $\Gamma_{\tau}^{\bar{V}^{\prime}}\left(p_{2}^{\prime} ; k^{\prime}\right)$ are transverse $\left(p_{1 \lambda}^{\prime} \Gamma_{\lambda}^{V}\left(p_{1}^{\prime} ; k\right)=p_{2 \tau}^{\prime} \Gamma_{\tau}^{\bar{V}^{\prime}}\left(p_{2}^{\prime} ; k^{\prime}\right)=0\right), \mathcal{N}^{V}$ and $\mathcal{N}^{\bar{V}^{\prime}}$ are the normalizations, and $\varphi_{V}\left(k^{2}\right)$ and $\varphi_{\bar{V}}\left(k^{2}\right)$ are scalar functions fixed by providing fits to the observables. The BS wave functions of vector mesons are [17]

$$
\begin{aligned}
\chi_{\lambda}\left(p_{1}^{\prime}, k\right)= & \frac{-1}{\gamma^{C} \cdot p_{1}-i m_{C}} \frac{1}{\mathcal{N}^{V}}\left(\gamma_{\lambda}+p_{1 \lambda}^{\prime} \frac{\gamma \cdot p_{1}^{\prime}}{M_{V}^{2}}\right) \\
& \times \varphi_{V}\left(k^{2}\right) \frac{-1}{\gamma^{A} \cdot p_{3}-i m_{A}}, \\
\chi_{\tau}\left(p_{2}^{\prime}, k^{\prime}\right)= & \frac{-1}{\gamma^{B} \cdot p_{4}-i m_{B}} \frac{1}{\mathcal{N}^{\bar{V}^{\prime}}}\left(\gamma_{\tau}+p_{2 \tau}^{\prime} \frac{\gamma \cdot p_{2}^{\prime}}{M_{\bar{V}^{\prime}}^{2}}\right) \\
& \times \varphi_{\bar{V}^{\prime}}\left(k^{\prime 2}\right) \frac{-1}{\gamma^{D} \cdot p_{2}-i m_{D}} .
\end{aligned}
$$

\section{GENERAL MATRIX ELEMENT BETWEEN FOUR-QUARK STATE AND VACUUM}

Because of the internal structure of the vector mesons in the molecular state, we investigate the photon interaction with quarks in vector mesons and consider that the quarks in a vector meson have different flavors in this work. The interaction Lagrangian for the coupling of quarks to photon is

$$
\begin{aligned}
\mathcal{L}_{I}\left(x_{i}\right)= & i \frac{n}{3} e \overline{\mathcal{Q}}\left(x_{i}\right) \gamma_{\mu} \mathcal{Q}\left(x_{i}\right) A_{\mu}\left(x_{i}\right) \\
& +i \frac{n^{\prime}}{3} e \overline{\mathcal{Q}}^{\prime}\left(x_{i}\right) \gamma_{\mu} \mathcal{Q}^{\prime}\left(x_{i}\right) A_{\mu}\left(x_{i}\right),
\end{aligned}
$$

where $e$ is the electron charge, $\frac{e^{2}}{4 \pi}=\frac{1}{137}$, the factors $n$ and $n^{\prime}$ are determined by the flavor of the quark, and $\mathcal{Q}$ and $\mathcal{Q}^{\prime}$ represent the quark field operators with different flavors. In Fig. 2, the radiative two-photon decay of molecular state is shown. The second-order S-matrix element between molecular state and two photons can be obtained,

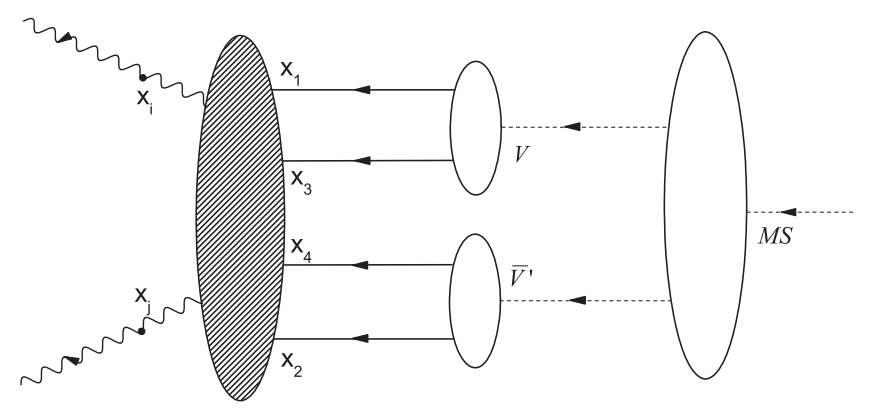

FIG. 2. General matrix element of the charge current between the four-quark state and vacuum in the coordinate representation. The filled ellipse represents the irreducible part of the Green's function.

$$
\begin{aligned}
& \left\langle\gamma \gamma\left|S^{(2)}\right| M S\right\rangle \\
& \quad=\frac{(-i)^{2}}{2 !} \int d^{4} x_{i} \int d^{4} x_{j}\left\langle\gamma \gamma\left|T \mathcal{H}_{I}\left(x_{i}\right) \mathcal{H}_{I}\left(x_{j}\right)\right| M S\right\rangle .
\end{aligned}
$$

Owing to Eq. (18), there should be two terms on the righthand side of Eq. (19), and these two terms are equal by the interchange of $x_{i}$ and $x_{j}$. Then, the lowest-order transition matrix element for the radiative two-photon decay of a molecular state composed of two vector mesons becomes

$$
\begin{aligned}
\left\langle\gamma \gamma\left|S^{(2)}\right| M S\right\rangle= & n n^{\prime}\left(\frac{e}{3}\right)^{2} \int d^{4} x_{i} \int d^{4} x_{j} \\
& \times\langle\gamma \gamma| T \overline{\mathcal{Q}}\left(x_{i}\right) \gamma_{\mu} \mathcal{Q}\left(x_{i}\right) A_{\mu}\left(x_{i}\right) \overline{\mathcal{Q}}^{\prime}\left(x_{j}\right) \\
& \times \gamma_{\nu} \mathcal{Q}^{\prime}\left(x_{j}\right) A_{\nu}\left(x_{j}\right)|M S\rangle .
\end{aligned}
$$

According to the S-matrix theory, we obtain

$$
\begin{aligned}
\left\langle\gamma \gamma\left|S^{(2)}\right| M S\right\rangle= & n n^{\prime}\left(\frac{e}{3}\right)^{2} \int d^{4} x_{i} d^{4} x_{j} \\
& \times \frac{1}{(2 \pi)^{3 / 2}} \frac{1}{\sqrt{2\left|\mathbf{Q}^{\prime}\right|}} \varepsilon_{\mu}^{Q^{\prime} *}\left(Q^{\prime}\right) \\
& \times e^{-i Q^{\prime} \cdot x_{i}} \frac{1}{(2 \pi)^{3 / 2}} \frac{1}{\sqrt{2|\mathbf{Q}|}} \varepsilon_{\nu}^{Q *}(Q) e^{-i Q \cdot x_{j}} \\
& \times\left\langle 0\left|T \overline{\mathcal{Q}}\left(x_{i}\right) \gamma_{\mu} \mathcal{Q}\left(x_{i}\right) \overline{\mathcal{Q}}^{\prime}\left(x_{j}\right) \gamma_{\nu} \mathcal{Q}^{\prime}\left(x_{j}\right)\right| P\right\rangle,
\end{aligned}
$$

where $Q$ and $Q^{\prime}$ are the momenta of two photons in the final state, $Q=(\mathbf{Q}, i|\mathbf{Q}|)$ and $Q^{\prime}=\left(\mathbf{Q}^{\prime}, i\left|\mathbf{Q}^{\prime}\right|\right)$, and $\varepsilon_{\nu}^{\varrho=1,2}(Q)$ and $\varepsilon_{\mu}^{\ell^{\prime}=1,2}\left(Q^{\prime}\right)$ are their polarization vectors, respectively.

Then, the charge current in Eq. (21) is $J_{\mu}\left(x_{i}\right) J_{\nu}\left(x_{j}\right)=$ $\overline{\mathcal{Q}}\left(x_{i}\right) \gamma_{\mu} \mathcal{Q}\left(x_{i}\right) \overline{\mathcal{Q}}^{\prime}\left(x_{j}\right) \gamma_{\nu} \mathcal{Q}^{\prime}\left(x_{j}\right)$. Applying Mandelstam's approach in quantum field theory [22], we obtain the general formulas for the matrix elements of charge current $J_{\mu} J_{\nu}$ between the four-quark state and vacuum 


$$
\begin{aligned}
& \left\langle 0\left|T J_{\mu}\left(x_{i}\right) J_{\nu}\left(x_{j}\right)\right| P\right\rangle \\
& =\int d^{4} x_{1} d^{4} x_{3} d^{4} x_{4} d^{4} x_{2} T\left(x_{i}, x_{j} ; x_{1}, x_{3}, x_{4}, x_{2}\right) \\
& \quad \times \chi_{P}^{j}\left(x_{1}, x_{3}, x_{4}, x_{2}\right),
\end{aligned}
$$

where $T\left(x_{i}, x_{j} ; x_{1}, x_{3}, x_{4}, x_{2}\right)$ is the irreducible part of the Green's function, shown as in Fig. 2. The filled ellipse in Fig. 2 represents the irreducible part of the Green's function, which is, in fact, a matrix element of a timeorder product of Heisenberg field operators. From the GBS wave function of the four-quark bound state defined in Eq. (1) and the charge current $J_{\mu} J_{\nu}$, it is straightforward to derive the two-particle irreducible Green's function

$$
\begin{aligned}
& T\left(x_{i}, x_{j} ; x_{1}, x_{3}, x_{4}, x_{2}\right) \\
& =\langle 0| T \overline{\mathcal{Q}}\left(x_{i}\right) \gamma_{\mu} \mathcal{Q}\left(x_{i}\right) \overline{\mathcal{Q}}^{\prime}\left(x_{j}\right) \gamma_{\nu} \mathcal{Q}^{\prime}\left(x_{j}\right) \\
& \quad \times \overline{\mathcal{Q}}^{C}\left(x_{1}\right) \mathcal{Q}^{A}\left(x_{3}\right) \overline{\mathcal{Q}}^{B}\left(x_{4}\right) \mathcal{Q}^{D}\left(x_{2}\right)|0\rangle_{T} .
\end{aligned}
$$

Since the electromagnetic interaction does not change the quark flavor, we obtain

$$
\begin{aligned}
T\left(x_{i},\right. & \left.x_{j} ; x_{1}, x_{3}, x_{4}, x_{2}\right) \\
= & \langle 0| T \overline{\mathcal{Q}}^{A}\left(x_{i}\right) \gamma_{\mu}^{A} \mathcal{Q}^{A}\left(x_{i}\right) \overline{\mathcal{Q}}^{C}\left(x_{j}\right) \gamma_{\nu}^{C} \mathcal{Q}^{C}\left(x_{j}\right) \\
& \times \overline{\mathcal{Q}}^{C}\left(x_{1}\right) \mathcal{Q}^{A}\left(x_{3}\right) \overline{\mathcal{Q}}^{A}\left(x_{4}\right) \mathcal{Q}^{C}\left(x_{2}\right)|0\rangle_{T}
\end{aligned}
$$

In quantum field theory, the matrix element in the Heisenberg picture cannot be calculated, so one has to transform it into the interaction picture, which can be analyzed in terms of perturbation theory. In the interaction picture, the two-particle irreducible Green's function becomes [22]

$$
\begin{aligned}
T\left(x_{i}, x_{j} ; x_{1}, x_{3}, x_{4}, x_{2}\right) \\
=\left(0 \mid T \overline{\mathcal{Q}}_{i p}^{A}\left(x_{i}\right) \gamma_{\mu}^{A} \mathcal{Q}_{i p}^{A}\left(x_{i}\right) \overline{\mathcal{Q}}_{i p}^{C}\left(x_{j}\right) \gamma_{\nu}^{C} \mathcal{Q}_{i p}^{C}\left(x_{j}\right)\right. \\
\left.\quad \times \overline{\mathcal{Q}}_{i p}^{C}\left(x_{1}\right) \mathcal{Q}_{i p}^{A}\left(x_{3}\right) \overline{\mathcal{Q}}_{i p}^{A}\left(x_{4}\right) \mathcal{Q}_{i p}^{C}\left(x_{2}\right) S \mid 0\right)_{T},
\end{aligned}
$$

where $\mathcal{Q}_{i p}$ represents the interaction picture field operator and $\mid 0)$ is the unperturbed free field vacuum. The operator $S$ in the right-hand side of Eq. (25) is

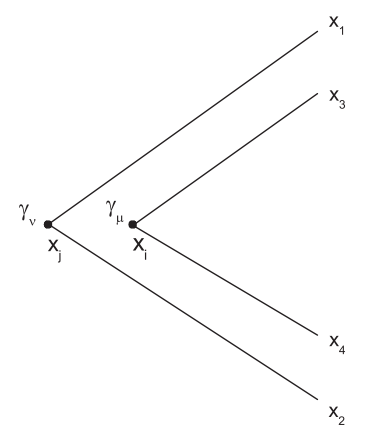

(a)

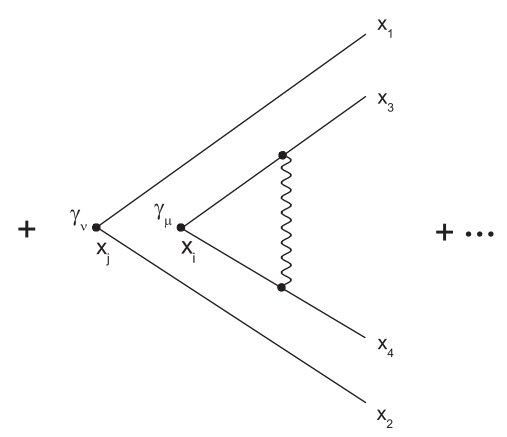

(b)
FIG. 3. Perturbation expansion of the two-particle irreducible Green's function. Diagram (a) represents the lowest-order term.

$$
S=T \exp \left[-i \int_{-\infty}^{\infty} d^{4} z \mathcal{H}_{I}^{i p}(z)\right]
$$

where $\mathcal{H}_{I}^{i p}(z)$ represents the interaction Hamiltonian density in the interaction picture. By means of perturbation theory, $T\left(x_{i}, x_{j} ; x_{1}, x_{3}, x_{4}, x_{2}\right)$ can be expressed as shown in Fig. 3.

In this work, we investigate the radiative decay of the molecular state. Because of the small electron charge, it is reasonable to consider that the lowest-order term of the irreducible part of the Green's function is dominant. The lowest-order term in the expansion of the right-hand side of Eq. (25) is shown as Fig. 3(a), and the lowest-order value of $T\left(x_{i}, x_{j} ; x_{1}, x_{3}, x_{4}, x_{2}\right)$ is

$$
\begin{aligned}
& T_{0}\left(x_{i}, x_{j} ; x_{1}, x_{3}, x_{4}, x_{2}\right) \\
& =\delta^{(4)}\left(x_{3}-x_{i}\right) \gamma_{\mu}^{A} \delta^{(4)}\left(x_{i}-x_{4}\right) \delta^{(4)}\left(x_{2}-x_{j}\right) \gamma_{\nu}^{C} \delta^{(4)}\left(x_{j}-x_{1}\right) .
\end{aligned}
$$

Substituting Eq. (27) into (22), we obtain the lowest-order matrix elements of $J_{\mu} J_{\nu}$ between the four-quark state and vacuum,

$$
\left\langle 0\left|T J_{\mu}\left(x_{i}\right) J_{\nu}\left(x_{j}\right)\right| P\right\rangle=\gamma_{\mu}^{A} \gamma_{\nu}^{C} \chi_{P}^{j}\left(x_{j}, x_{i}, x_{i}, x_{j}\right) .
$$

From Eq. (3), the result (28) can be transformed to

$$
\begin{aligned}
\left\langle 0\left|T J_{\mu}\left(x_{i}\right) J_{\nu}\left(x_{j}\right)\right| P\right\rangle= & \frac{1}{(2 \pi)^{16}} \int d^{4} p_{1} d^{4} p_{3} d^{4} p_{4} d^{4} p_{2} \gamma_{\mu}^{A} \gamma_{\nu}^{C} \chi_{P}^{j}\left(p_{1}, p_{3}, p_{4}, p_{2}\right) e^{i p_{1} \cdot x_{j}} e^{i p_{3} \cdot x_{i}} e^{i p_{4} \cdot x_{i}} e^{i p_{2} \cdot x_{j}} \\
= & \frac{1}{(2 \pi)^{12}} \frac{1}{(2 \pi)^{3 / 2}} \frac{1}{\sqrt{2 E(P)}} \int d^{4} p_{1} d^{4} p_{3} d^{4} p_{4} d^{4} p_{2} \gamma_{\mu}^{A} \gamma_{\nu}^{C} \\
& \times \delta^{(4)}\left(P-p_{1}-p_{3}-p_{4}-p_{2}\right) \chi^{j}\left(P, p, k, k^{\prime}\right) e^{i\left(p_{1}+p_{2}\right) \cdot x_{j}} e^{i\left(p_{3}+p_{4}\right) \cdot x_{i}},
\end{aligned}
$$

where $\chi^{j}\left(P, p, k, k^{\prime}\right)$ is the GBS wave function for the four-quark state expressed as Eq. (7), $p_{1}=\eta_{1}^{\prime \prime}\left(\eta_{1} P+p\right)+k$, $p_{2}=\left(\eta_{2} P-p\right)-\left[P-Q-\eta_{3}^{\prime \prime}\left(\eta_{1} P+p\right)+k\right], \quad p_{3}=\eta_{3}^{\prime \prime}\left(\eta_{1} P+p\right)-k, \quad p_{4}=P-Q-\eta_{3}{ }^{\prime \prime}\left(\eta_{1} P+p\right)+k \quad$ and $\quad k^{\prime}=$ $\eta_{4}{ }^{\prime \prime}\left(\eta_{2} P-p\right)-\left[P-Q-\eta_{3}{ }^{\prime \prime}\left(\eta_{1} P+p\right)+k\right]$. 
Using the matrix elements of $J_{\mu} J_{\nu}$ between the four-quark state and vacuum in Eq. (29), we can obtain the computable form of the S-matrix element between the molecular state and two photons in the momentum representation,

$$
\begin{aligned}
\left\langle\gamma \gamma\left|S^{(2)}\right| M S\right\rangle= & n n^{\prime}\left(\frac{e}{3}\right)^{2} \frac{1}{(2 \pi)^{3 / 2}} \frac{1}{\sqrt{2\left|\mathbf{Q}^{\prime}\right|}} \frac{1}{\sqrt{2|\mathbf{Q}|}} \frac{1}{\sqrt{2 E(P)}} \frac{1}{(2 \pi)^{3}} \varepsilon_{\mu}^{e^{\prime *}}\left(Q^{\prime}\right) \varepsilon_{\nu} \varepsilon^{* *}(Q) \\
& \times(2 \pi)^{4} \delta^{(4)}\left(P-Q-Q^{\prime}\right) \int d^{4} k d^{4} p \frac{1}{(2 \pi)^{8}} \gamma_{\mu}^{A} \gamma_{\nu}^{C} \chi^{j}\left(P, p, k, k^{\prime}\right) .
\end{aligned}
$$

This matrix element is represented graphically in Fig. 4. It is necessary to note that the two-photon decay of a molecular state has two possibilities by the interchange of two final photons, and we find that the matrix element in Eq. (30) is invariant under the substitutions $Q \rightleftharpoons Q^{\prime}$ and $\varepsilon^{Q}(Q) \rightleftharpoons \varepsilon^{Q^{\prime}}\left(Q^{\prime}\right)$. Therefore, the total matrix element for the radiative two-photon decay of the molecular state composed of two vector mesons is

$$
\left\langle\gamma \gamma\left|S_{\text {tot }}^{(2)}\right| M S\right\rangle=2\left\langle\gamma \gamma\left|S^{(2)}\right| M S\right\rangle
$$

\section{DECAY MODE $X(3915) \rightarrow \gamma \gamma$}

As an application, we investigate the radiative twophoton decay of the exotic state $X(3915)$ in this section. Here, the isoscalar $X(3915)$, once named $Y(3940)$, is considered as a mixed state of two $\mathrm{S}$-wave molecule states $D^{* 0} \bar{D}^{* 0}$ and $D^{*+} D^{*-}$. The BS wave function of the $X(3915)$ state is a linear combination of these two components as

$\chi_{\lambda \tau}^{X(3915), j}(P, p)=\frac{1}{\sqrt{2}} \chi_{\lambda \tau}^{D^{* 0} \bar{D}^{* 0}, j}(P, p)+\frac{1}{\sqrt{2}} \chi_{\lambda \tau}^{D^{*+} D^{*-, j}}(P, p)$,

where

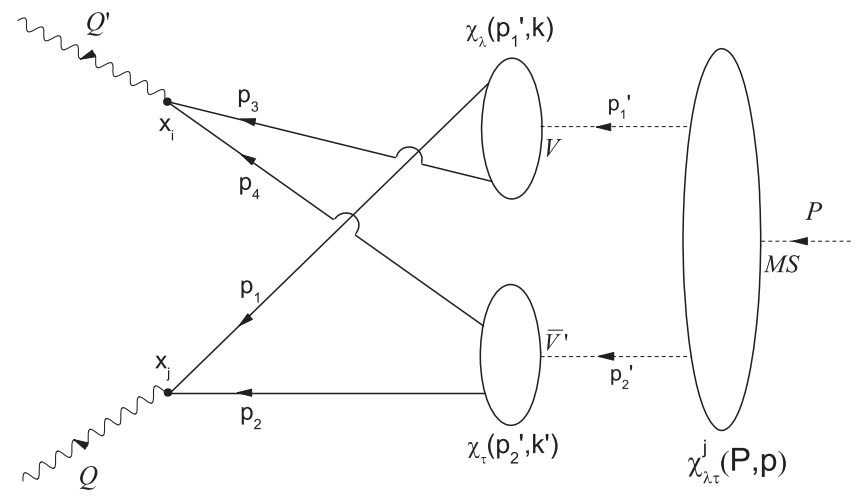

FIG. 4. The lowest-order matrix element between the fourquark state and vacuum in the momentum representation.

$$
\begin{aligned}
& \left.\chi_{\lambda \tau}^{D^{* 0} \bar{D}^{* 0}, j}(P, p)=\chi_{\lambda \tau}^{j}(P, p)\left(-\left|\frac{1}{2},-\frac{1}{2}\right\rangle\right)\right)^{D^{* 0}} \otimes\left|\frac{1}{2}, \frac{1}{2}\right\rangle^{\bar{D}^{* 0}}, \\
& \chi_{\lambda \tau}^{D^{*+} D^{*-}, j}(P, p)=\chi_{\lambda \tau}^{j}(P, p)\left|\frac{1}{2}, \frac{1}{2}\right\rangle^{D^{*+}} \otimes\left(-\left|\frac{1}{2},-\frac{1}{2}\right\rangle\right)^{D^{*-}},
\end{aligned}
$$

and $P$ becomes the total momentum of the $X(3915)$ state and $\chi_{\lambda \tau}^{j}(P, p)$ is the component wave function in the momentum representation; $\left(-\left|\frac{1}{2},-\frac{1}{2}\right\rangle\right) \otimes\left|\frac{1}{2}, \frac{1}{2}\right\rangle$ and $\left|\frac{1}{2}, \frac{1}{2}\right\rangle \otimes\left(-\left|\frac{1}{2},-\frac{1}{2}\right\rangle\right)$ are the isospin wave functions of pure molecule states $D^{* 0} \bar{D}^{* 0}$ and $D^{*+} D^{*-}$, respectively; and $\chi_{\lambda \tau}^{D^{\star 0} D^{* 0}, j}$ and $\chi_{\lambda \tau}^{D^{*+} D^{*-}, j}$ represent the BS wave functions for the bound states of two vector mesons, which are the eigenstates of the Hamiltonian without considering the coupled-channel terms. These eigenstates have the same quantum numbers. Since the $C$ parity of $X(3915)$ is even, one can have $J^{P}=0^{+}$or $2^{+}$for this S-wave $D^{*} \bar{D}^{*}$ system [23]. In Ref. [7], we only considered the neutral component $D^{* 0} \bar{D}^{* 0}$ to evaluate the mass and BS wave function of the molecular state. In this paper, we reconsider the mixed states of neutral component $D^{* 0} \bar{D}^{* 0}$ and charged component $D^{*+} D^{*-}$ with $J^{P}=0^{+}$and $2^{+}$.

For the neutral component, $V$ and $\bar{V}^{\prime}$ in Fig. 4 represent $D^{* 0}$ and $\bar{D}^{* 0}$, respectively; the flavor labels $A=B$ and $C=D$ in Eqs. (17) and (30) represent the $u$ quark and $c$ quark, respectively. For the charged component, $V$ and $\bar{V}^{\prime}$ become $D^{*+}$ and $D^{*-}$, respectively; the flavor labels $A=B$ and $C=D$ become the $d$ quark and $c$ quark, respectively. According to the Weinberg-Salam model, the interaction Lagrangian for the coupling of quarks to photon becomes

$$
\begin{aligned}
\mathcal{L}_{I}^{e m}\left(x_{i}\right)= & i e\left[\frac{2}{3} \bar{u}\left(x_{i}\right) \gamma_{\mu} u\left(x_{i}\right)-\frac{1}{3} \bar{d}\left(x_{i}\right) \gamma_{\mu} d\left(x_{i}\right)\right. \\
& \left.-\frac{1}{3} \bar{s}\left(x_{i}\right) \gamma_{\mu} s\left(x_{i}\right)+\frac{2}{3} \bar{c}\left(x_{i}\right) \gamma_{\mu} c\left(x_{i}\right)\right] A_{\mu}\left(x_{i}\right) .
\end{aligned}
$$

Then, the lowest-order matrix element for this decay process becomes 


$$
\begin{aligned}
\left\langle\gamma \gamma\left|S_{\mathrm{tot}}^{(2)}\right| X(3915)\right\rangle= & \frac{2}{\sqrt{2}} \frac{1}{(2 \pi)^{3 / 2}} \frac{1}{\sqrt{2\left|\mathbf{Q}^{\prime}\right|}} \frac{1}{\sqrt{2|\mathbf{Q}|}} \frac{1}{\sqrt{2 E(P)}} \frac{1}{(2 \pi)^{3}} \varepsilon_{\mu}^{Q^{\prime *}}\left(Q^{\prime}\right) \varepsilon_{\nu}^{Q *}(Q)(2 \pi)^{4} \delta^{(4)}\left(P-Q-Q^{\prime}\right) \\
& \times \int d^{4} k d^{4} p \frac{1}{(2 \pi)^{8}} \gamma_{\mu}^{A} \gamma_{\nu}^{C}\left[\frac{4 e^{2}}{9} \chi_{D^{* 0} \bar{D}^{* 0}}^{j}\left(P, p, k, k^{\prime}\right)-\frac{2 e^{2}}{9} \chi_{D^{*+} D^{*-}}^{j}\left(P, p, k, k^{\prime}\right)\right] .
\end{aligned}
$$

Let $D_{l}^{*}$ denote one of $D^{* 0}$ and $D^{*+}$, and $l=u, d$ represents the $u$ or $d$ antiquark in the heavy vector meson $D^{* 0}$ or $D^{*+}$, respectively; $\bar{D}_{l}^{*}$ denotes the antiparticle of $D_{l}^{*}$. From Eqs. (7) and (17), we obtain the GBS wave function for the four-quark state $[9,24]$

$$
\begin{aligned}
\chi_{D_{l}^{*} \bar{D}_{l}^{*}}^{j}\left(P, p, k, k^{\prime}\right)= & (2 \pi)^{8} \frac{-1}{\gamma^{C} \cdot p_{1}-i m_{c}} \frac{1}{\mathcal{N}^{D_{l}^{*}}}\left(\gamma_{\lambda}+p_{1 \lambda}^{\prime} \frac{\gamma \cdot p_{1}^{\prime}}{M_{D_{l}^{*}}^{2}}\right) \varphi_{D_{l}^{*}}\left(k^{2}\right) \frac{-1}{\gamma^{A} \cdot p_{3}-i m_{l}} \chi_{\lambda \tau}^{j}(P, p) \\
& \times \frac{-1}{\gamma^{A} \cdot p_{4}-i m_{l}} \frac{1}{\mathcal{N}^{\bar{D}_{l}^{*}}}\left(\gamma_{\tau}+p_{2 \tau}^{\prime} \frac{\gamma \cdot p_{2}^{\prime}}{M_{\bar{D}_{l}^{*}}^{2}}\right) \varphi_{\bar{D}_{l}^{*}}\left(k^{\prime 2}\right) \frac{-1}{\gamma^{C} \cdot p_{2}-i m_{c}},
\end{aligned}
$$

where $m_{c, l}$ are the constituent quark masses, $\varphi_{D_{l}^{*}}\left(k^{2}\right)=$ $\varphi_{\bar{D}_{l}^{*}}\left(k^{2}\right)=\exp \left(-k^{2} / \omega_{D^{*}}^{2}\right), \omega_{D^{*}}=1.50 \mathrm{GeV}[21]$, and the momentum of the $X(3915)$ state is set as $P=(0,0,0, i M)$ in the rest frame. As in heavy-quark effective theory [25], we consider that the heaviest quark carries all the heavymeson momentum, and these momenta in Eq. (29) become

$$
\begin{aligned}
& p_{1}=P / 2+p+k, \quad p_{2}=Q-P / 2-p-k, \\
& p_{3}=-k, \quad p_{4}=-k^{\prime}, \\
& p_{1}^{\prime}=P / 2+p, \quad p_{2}^{\prime}=P / 2-p, \quad k^{\prime}=Q-P-k .
\end{aligned}
$$

Now, we determine the normalizations $\mathcal{N}^{D^{* 0}}$ and $\mathcal{N}^{D^{*+}}$. The component wave functions $\chi_{\lambda \tau}^{j}(P, p)$ with $J^{P}=0^{+}$ and $2^{+}$are given in Secs. IV. A and IV. B, respectively. From Eq. (17), the BS wave function of the $D_{l}^{*}$ meson can be written as

$$
\begin{aligned}
\chi_{\lambda}(K, k)= & \frac{-1}{\gamma \cdot(K+k)-i m_{c}} \frac{1}{\mathcal{N}^{D_{l}^{*}}}\left(\gamma_{\lambda}+K_{\lambda} \frac{\gamma \cdot K}{M_{D_{l}^{*}}^{2}}\right) \varphi_{D_{l}^{*}}\left(k^{2}\right) \\
& \times \frac{-1}{\gamma \cdot(-k)-i m_{l}},
\end{aligned}
$$

where $K$ is set as the momentum of the heavy meson in the rest frame, $k$ denotes the relative momentum between the $c$ quark and $l$ antiquark, and $k$ and $K$ are not the momenta presented in the decay process. For $l=u, \mathcal{N}^{D_{l}^{*}}=\mathcal{N}^{D^{* 0}}$ represents the normalization of the BS wave function for the heavy vector meson $D^{* 0}$; for $l=d, \mathcal{N}^{D_{l}^{*}}=\mathcal{N}^{D^{*+}}$ represents the normalization of the $\mathrm{BS}$ wave function for the heavy vector meson $D^{*+}$. The authors of Refs. [20,21] employed the ladder approximation to solve the BS equation for quark-antiquark state, and the reduced normalization condition for the BS wave function of $D_{l}^{*}$ meson given by Eq. (38) is

$$
\begin{aligned}
2 K_{0}= & \frac{-i}{(2 \pi)^{4}} \frac{1}{3} \int d^{4} k \bar{\chi}_{\lambda}(K, k) \\
& \times \frac{\partial}{\partial K_{0}}\left[S(K+k)^{-1}\right] S(-k)^{-1} \chi_{\lambda}(K, k),
\end{aligned}
$$

where the factor $1 / 3$ appears because of the sum of three transverse directions.

\section{A. Molecular state with $J^{P}=0^{+}$}

In Ref. [7], the BS equation for the bound state composed of two heavy vector mesons was treated in the ladder approximation, and the BS wave function of pure molecule state $D_{l}^{*} \bar{D}_{l}^{*}$ with $0^{+}$was obtained:

$$
\begin{aligned}
& \chi_{\lambda \tau}^{0^{+}}\left(P^{D \bar{D}}, p\right) \\
& =\frac{1}{\mathcal{N}_{D \bar{D}}^{0^{+}}}\left[T_{\lambda \tau}^{1} \mathcal{F}_{1}\left(P^{D \bar{D}} \cdot p, p^{2}\right)+T_{\lambda \tau}^{2} \mathcal{F}_{2}\left(P^{D \bar{D}} \cdot p, p^{2}\right)\right] .
\end{aligned}
$$

$P^{D \bar{D}}$ represents the momentum of the pure molecule state in the rest frame, the fourth component of which is different from the one of $P$. These scalar functions $\mathcal{F}_{1}$ and $\mathcal{F}_{2}$ should satisfy two individual equations [7]

$$
\mathcal{F}_{1}\left(P^{D \bar{D}} \cdot p, p^{2}\right)=\frac{1}{\left(P^{D \bar{D}} / 2+p\right)^{2}+M_{1}^{2}-i \epsilon} \frac{1}{\left(P^{D \bar{D}} / 2-p\right)^{2}+M_{2}^{2}-i \epsilon} \int \frac{d^{4} q^{\prime}}{(2 \pi)^{4}} V_{1}\left(p, q^{\prime} ; P^{D \bar{D}}\right) \mathcal{F}_{1}\left(P^{D \bar{D}} \cdot q^{\prime}, q^{\prime 2}\right)
$$




$$
\begin{aligned}
\left(P^{D \bar{D}} / 2-p\right)^{2} \mathcal{F}_{2}\left(P^{D \bar{D}} \cdot p, p^{2}\right)= & \frac{1}{\left(P^{D \bar{D}} / 2+p\right)^{2}+M_{1}^{2}-i \epsilon} \frac{1}{\left(P^{D \bar{D}} / 2-p\right)^{2}+M_{2}^{2}-i \epsilon} \\
& \times \int \frac{d^{4} q^{\prime}}{(2 \pi)^{4}} V_{2}\left(p, q^{\prime} ; P^{D \bar{D}}\right)\left(P^{D \bar{D}} / 2-q^{\prime}\right)^{2} \mathcal{F}_{2}\left(P^{D \bar{D}} \cdot q^{\prime}, q^{\prime 2}\right),
\end{aligned}
$$

where $V_{1}\left(p, q^{\prime} ; P^{D \bar{D}}\right)$ and $V_{2}\left(p, q^{\prime} ; P^{D \bar{D}}\right)$ are derived from the interaction kernel between $D_{l}^{*} \bar{D}_{l}^{*}$. Considering the internal structure of vector meson $D_{l}^{*}$ and $\mathrm{SU}(3)$ symmetry, we constructed the interaction kernel between two vector mesons derived from one light meson $\left(\sigma, \omega, \rho^{0}, \phi\right)$ exchange, shown as Eq. (35) in Ref. [7]. To avoid confusion, the momentum of the exchanged meson is denoted by $a$ in this paper. In instantaneous approximation, we set the momentum of the exchanged meson as $a=(\boldsymbol{a}, 0)$; Eqs. (41) and (42) in this paper become two relativistic Schrödinger-like equations shown as Eqs. (41) and (44) in Ref. [7], respectively. Solving these two equations, we obtained the wave functions $\Psi_{1}^{0^{+}}(\mathbf{p})=\int d p_{0} \mathcal{F}_{1}\left(P^{D \bar{D}} \cdot p, p^{2}\right)$ and $\Psi_{2}^{0^{+}}(\mathbf{p})=$ $\int d p_{0}\left(P^{D \bar{D}} / 2-p\right)^{2} \mathcal{F}_{2}\left(P^{D \bar{D}} \cdot p, p^{2}\right)$ in Ref. [7]. And then, the masses and wave functions of pure molecule states $D^{* 0} \bar{D}^{* 0}$ and $D^{*+} D^{*-}$ with $J^{P}=0^{+}$can be obtained.

Considering one light meson $\left(\rho^{+}\right.$and $\left.\rho^{-}\right)$exchange, we obtained the cross-terms between two pure molecule states $D^{* 0} \bar{D}^{* 0}$ and $D^{*+} D^{*-}$, shown as the first term of Eq. (16) in Ref. [8]. And then, the matrix elements between two pure molecule states were obtained in the instantaneous approximation shown as Eq. (43) in Ref. [8]. Using the coupledchannel approach (see details in Ref. [8]), we can calculate the mass of the $X(3915)$ state with $J^{P}=0^{+}$. Since the mixing of component wave functions causes the change of energy, the fourth component of $P^{D \bar{D}}$ in the original BS wave function becomes the total energy of mixed state, and $\chi_{\lambda \tau}^{0^{+}}\left(P^{D \bar{D}}, p\right)$ in Eq. (40) becomes

$$
\chi_{\lambda \tau}^{0^{+}}(P, p)=\frac{1}{\mathcal{N}^{0^{+}}}\left[T_{\lambda \tau}^{1} \mathcal{F}_{1}\left(P \cdot p, p^{2}\right)+T_{\lambda \tau}^{2} \mathcal{F}_{2}\left(P \cdot p, p^{2}\right)\right] .
$$

The reduced normalization condition for $\chi_{\lambda \tau}^{0^{+}}(P, p)$ is

$$
\begin{aligned}
2 P_{0}= & \frac{-i}{(2 \pi)^{4}} \int d^{4} p \bar{\chi}_{\lambda \tau}(P, p) \\
& \times \frac{\partial}{\partial P_{0}}\left[\Delta_{F \lambda \lambda^{\prime}}(P / 2+p)^{-1} \Delta_{F \tau \tau^{\prime}}(P / 2-p)^{-1}\right] \chi_{\lambda^{\prime} \tau^{\prime}}(P, p),
\end{aligned}
$$

where $\Delta_{F \beta \alpha^{\prime}}(p)^{-1}$ is the inverse propagator for the vector field with mass $m, \Delta_{F \beta \alpha^{\prime}}(p)^{-1}=i\left(\delta_{\beta \alpha^{\prime}}-\frac{p_{\beta} p_{\alpha^{\prime}}}{p^{2}+m^{2}}\right)\left(p^{2}+m^{2}\right)$ [9]. To fix the normalization $\mathcal{N}^{0^{+}}$, we require the scalar functions $\mathcal{F}_{1}$ and $\mathcal{F}_{2}$, which can be obtained from $\Psi_{1}^{0^{+}}$and $\Psi_{2}^{0^{+}}$, respectively. After determining the normalization $\mathcal{N}^{0^{+}}$, we automatically obtain the normalized BS wave function for the mixed state of two components $D^{* 0} \bar{D}^{* 0}$ and $D^{*+} D^{*-}$ given by Eq. (32).

Substituting Eq. (43) into (36), we obtain the GBS wave function for four-quark state $\chi_{D_{D}^{*} \bar{D}_{l}^{*}}^{0}\left(P, p, k, k^{\prime}\right)$ with $J^{P}=0^{+}$. Then, the lowest-order transition matrix element for the radiative two-photon decay of $X(3915)$ expressed as Eq. (35) can be calculated,

$$
\begin{aligned}
\left\langle\gamma \gamma\left|S_{\mathrm{tot}}^{(2)}\right| X(3915)\right\rangle= & \frac{2}{\sqrt{2}} \frac{1}{(2 \pi)^{3 / 2}} \frac{1}{\sqrt{2\left|\mathbf{Q}^{\prime}\right|}} \frac{1}{\sqrt{2|\mathbf{Q}|}} \frac{1}{\sqrt{2 E(P)}} \frac{1}{(2 \pi)^{3}} \varepsilon_{\mu}^{Q^{\prime} *}\left(Q^{\prime}\right) \varepsilon_{\nu}^{Q *}(Q) \\
& \times(2 \pi)^{4} \delta^{(4)}\left(P-Q-Q^{\prime}\right)\left(\frac{4 e^{2}}{9} \mathcal{M}_{\nu \mu}^{D^{* *} \bar{D}^{* 0}}-\frac{2 e^{2}}{9} \mathcal{M}_{\nu \mu}^{D^{*+} D^{*-}}\right),
\end{aligned}
$$

where

$$
\begin{aligned}
\mathcal{M}_{\nu \mu}^{D_{l}^{*} \bar{D}_{l}^{*}}= & \int d^{4} k d^{4} p \frac{1}{p_{1}^{2}+m_{c}^{2}} \frac{1}{\mathcal{N}^{D_{l}^{*}}} \frac{\varphi_{D_{l}^{*}}\left(k^{2}\right)}{k^{2}+m_{l}^{2}} \frac{1}{\mathcal{N}^{\bar{D}_{l}^{*}}} \frac{\varphi_{\bar{D}_{l}^{*}}\left(k^{\prime 2}\right)}{k^{\prime 2}+m_{l}^{2}} \frac{1}{p_{2}^{2}+m_{c}^{2}} \frac{1}{\mathcal{N}^{0^{+}}} \\
& \times \operatorname{Tr}\left\{\gamma _ { \nu } [ \gamma \cdot p _ { 1 } + i m _ { c } ] \left\{\left[\left(p_{1}^{\prime} \cdot p_{2}^{\prime}\right) \gamma_{\tau}-\left(\gamma \cdot p_{2}^{\prime}\right) p_{1 \tau}^{\prime}\right] \mathcal{F}_{1}\left(P \cdot p, p^{2}\right)+\left[p_{1}^{\prime 2} p_{2}^{\prime 2} \gamma_{\tau}\right.\right.\right. \\
& \left.\left.+\left(p_{1}^{\prime} \cdot p_{2}^{\prime}\right)\left(\gamma \cdot p_{1}^{\prime}\right) p_{2 \tau}^{\prime}-p_{2}^{\prime 2}\left(\gamma \cdot p_{1}^{\prime}\right) p_{1 \tau}^{\prime}-p_{1}^{\prime 2}\left(\gamma \cdot p_{2}^{\prime}\right) p_{2 \tau}^{\prime}\right] \mathcal{F}_{2}\left(P \cdot p, p^{2}\right)\right\} \\
& \left.\times\left[\gamma \cdot(-k)+i m_{l}\right] \gamma_{\mu}\left[\gamma \cdot\left(-k^{\prime}\right)+i m_{l}\right] \gamma_{\tau}\left[\gamma \cdot p_{2}+i m_{c}\right]\right\} .
\end{aligned}
$$


In Eq. (46), the trace of the product of eight $\gamma$-matrices contains 105 terms, and the resulting expression has been given in Appendix B of Ref. [9]. In our approach, the $p$ integral is computed in the instantaneous approximation. To calculate this tensor $\mathcal{M}_{\nu \mu}^{D_{l}^{*} \bar{D}_{l}^{*}}$, we have given a simple method in Ref. [9]. It is obvious that the tensor $\mathcal{M}_{\nu \mu}^{D_{\mu}^{*} \bar{D}_{l}^{*}}$ only depends on $P$ and $Q$, so in Minkowski space, $\mathcal{M}_{\nu \mu}^{D_{D}^{*} \bar{D}_{l}^{*}}$ can be expressed as

$$
\begin{aligned}
\mathcal{M}_{\nu \mu}^{D_{\nu}^{*} \bar{D}_{l}^{*}}= & g_{\nu \mu} U_{1}(P, Q)+P_{\nu} Q_{\mu} U_{2}(P, Q)+P_{\nu} P_{\mu} U_{3}(P, Q) \\
& +Q_{\nu} P_{\mu} U_{4}(P, Q)+Q_{\nu} Q_{\mu} U_{5}(P, Q),
\end{aligned}
$$

where $U_{i}(P, Q)(i=1, \ldots, 5)$ are the scalar functions. The above expression is multiplied by these tensor structures $g_{\nu \mu}, P_{\nu} Q_{\mu}, P_{\nu} P_{\mu}, Q_{\nu} P_{\mu}$, and $Q_{\nu} Q_{\mu}$, respectively, and a set of equations is obtained,

$$
\begin{aligned}
g_{\nu \mu} \mathcal{M}_{\nu \mu}^{D_{l}^{*} \bar{D}_{l}^{*}}=U_{1}^{\prime}=4 U_{1}+(P \cdot Q) U_{2}+P^{2} U_{3}+(P \cdot Q) U_{4}+Q^{2} U_{5}, \\
P_{\nu} Q_{\mu} \mathcal{M}_{\nu \mu}^{D_{l}^{*} \bar{D}_{l}^{*}}=U_{2}^{\prime}=(P \cdot Q) U_{1}+P^{2} Q^{2} U_{2}+P^{2}(P \cdot Q) U_{3}+(P \cdot Q)^{2} U_{4}+Q^{2}(P \cdot Q) U_{5}, \\
P_{\nu} P_{\mu} \mathcal{M}_{\nu \mu}^{D_{l}^{*} \bar{D}_{l}^{*}}=U_{3}^{\prime}=P^{2} U_{1}+P^{2}(P \cdot Q) U_{2}+P^{2} P^{2} U_{3}+P^{2}(P \cdot Q) U_{4}+(P \cdot Q)^{2} U_{5}, \\
Q_{\nu} P_{\mu} \mathcal{M}_{\nu \mu}^{D_{l}^{*} \bar{D}_{l}^{*}}=U_{4}^{\prime}=(P \cdot Q) U_{1}+(P \cdot Q)^{2} U_{2}+P^{2}(P \cdot Q) U_{3}+Q^{2} P^{2} U_{4}+Q^{2}(P \cdot Q) U_{5}, \\
Q_{\nu} Q_{\mu} \mathcal{M}_{\nu \mu}^{D_{l}^{*} \bar{D}_{l}^{*}}=U_{5}^{\prime}=Q^{2} U_{1}+Q^{2}(P \cdot Q) U_{2}+(P \cdot Q)^{2} U_{3}+Q^{2}(P \cdot Q) U_{4}+Q^{2} Q^{2} U_{5},
\end{aligned}
$$

where $U_{i}^{\prime}$ are numbers. Subsequently, we numerically calculate $U_{i}^{\prime}$ and solve this set of equations. The values of $U_{i}$ can be obtained.

Finally, we obtain the radiative two-photon decay width of $X(3915)$ with $J^{P}=0^{+}$,

$$
\begin{aligned}
\Gamma^{0^{+}}= & \int d^{3} Q d^{3} Q^{\prime}(2 \pi)^{4} \delta^{(4)}\left(P-Q-Q^{\prime}\right) 2 \frac{1}{2\left|\mathbf{Q}^{\prime}\right|} \frac{1}{2|\mathbf{Q}|} \frac{1}{2 E(P)} \frac{1}{(2 \pi)^{6}} \\
& \times \sum_{\varrho^{\prime}=1}^{2} \sum_{\varrho=1}^{2}\left|\varepsilon_{\mu}^{\ell^{\prime}}\left(Q^{\prime}\right) \varepsilon_{\nu}^{\varrho}(Q)\left(\frac{4 e^{2}}{9} \mathcal{M}_{\nu \mu}^{D^{* 0} D^{* 0}}-\frac{2 e^{2}}{9} \mathcal{M}_{\nu \mu}^{D^{*+} D^{*-}}\right)\right|^{2},
\end{aligned}
$$

where $P=(0,0,0, i M), Q=\left(\mathbf{Q}_{\gamma}, i\left|\mathbf{Q}_{\gamma}\right|\right), Q^{\prime}=\left(-\mathbf{Q}_{\gamma}, i\left|\mathbf{Q}_{\gamma}\right|\right)$, and $\left|\mathbf{Q}_{\gamma}\right|=M / 2$. To derive Eq. (49), we use the transverse condition of electromagnetic field $\varepsilon^{Q}(Q) \cdot Q=0$ and the completeness relation.

\section{B. Molecular state with $\mathbf{J}^{P}=2^{+}$}

Because we consider the S-wave molecule state composed of $D_{l}^{*} \bar{D}_{l}^{*}$ with $J^{P}=2^{+}$, the tensor structure in its BS wave function cannot contain the relative momenta $p_{\mu_{1}}, \ldots, p_{\mu_{j}}$. This is because the relative momentum represents the orbital angular momentum. In Eq. (13), only one term describes the S-wave vector-vector system,

$$
\begin{aligned}
\chi_{\lambda \tau}^{2_{\lambda}^{+}}\left(P^{D \bar{D}}, p\right) & =\frac{1}{\mathcal{N}_{D \bar{D}}^{2^{+}}} \eta_{\mu_{1} \mu_{2}}^{\varsigma} g_{\mu_{1} \lambda} g_{\mu_{2} \tau} \mathcal{G}_{5}\left(P^{D \bar{D}} \cdot p, p^{2}\right) \\
& =\frac{1}{\mathcal{N}_{D \bar{D}}^{2+}} \eta_{\lambda \tau}^{\varsigma} \mathcal{G}_{5}\left(P^{D \bar{D}} \cdot p, p^{2}\right)
\end{aligned}
$$

where $\eta_{\lambda \tau}^{\varsigma=1,2,3,4,5}$ is the polarization tensor of the molecular state with $J^{P}=2^{+}$. In Ref. [7], Eq. (22) is wrong, it is revised as (50) in this paper. This BS wave function should satisfy the equation

$$
\begin{aligned}
\chi_{\lambda \tau}^{2_{\lambda}^{+}}\left(P^{D \bar{D}}, p\right)= & -\int \frac{d^{4} q^{\prime}}{(2 \pi)^{4}} \Delta_{F \lambda \alpha}\left(p_{1}^{\prime}\right) \mathcal{V}_{\alpha \theta, \beta \kappa}\left(p, q^{\prime} ; P^{D \bar{D}}\right) \\
& \times \chi_{\theta \kappa}^{2^{+}}\left(P^{D \bar{D}}, q^{\prime}\right) \Delta_{F \beta \tau}\left(p_{2}^{\prime}\right),
\end{aligned}
$$

where $\mathcal{V}_{\alpha \theta, \beta \kappa}$ is the interaction kernel and $\Delta_{F \lambda \alpha}\left(p_{1}^{\prime}\right)$ and $\Delta_{F \beta \tau}\left(p_{2}^{\prime}\right)$ are the propagators for the spin 1 fields. The interaction kernel between $D_{l}^{*}$ and $\bar{D}_{l}^{*}$ derived from one light meson $\left(\sigma, \omega, \rho^{0}, \phi\right)$ exchange was constructed in Ref. [7]. Comparing the terms $\eta_{\lambda \tau}^{\varsigma}$ in the left and right sides of Eq. (51), we obtain

$$
\begin{aligned}
\mathcal{G}_{5}\left(P^{D \bar{D}} \cdot p, p^{2}\right)= & \frac{1}{\left(P^{D \bar{D}} / 2+p\right)^{2}+M_{1}^{2}-i \epsilon} \\
& \times \frac{1}{\left(P^{D \bar{D}} / 2-p\right)^{2}+M_{2}^{2}-i \epsilon} \\
& \times \int \frac{d^{4} q^{\prime}}{(2 \pi)^{4}} V_{5}\left(p, q^{\prime} ; P^{D \bar{D}}\right) \mathcal{G}_{5}\left(P^{D \bar{D}} \cdot q^{\prime}, q^{\prime 2}\right),
\end{aligned}
$$

where $V_{5}\left(p, q^{\prime} ; P^{D \bar{D}}\right)$ contains all coefficients of the term $\eta_{\lambda \tau}^{\varsigma}$ in the right side of (51). Setting $\Psi^{2^{+}}(\mathbf{p})=\int d p_{0} \mathcal{G}_{5}\left(P^{D \bar{D}}\right.$. $p, p^{2}$ ) and adopting instantaneous approximation, we obtain the Schrödinger-type equation (see details in Ref. [7]) 


$$
\left(\frac{b^{2}(M)}{2 \mu_{R}}-\frac{\mathbf{p}^{2}}{2 \mu_{R}}\right) \Psi^{2^{+}}(\mathbf{p})=\int \frac{d^{3} a}{(2 \pi)^{3}} V^{2^{+}}(\mathbf{p}, \boldsymbol{a}) \Psi^{2^{+}}(\mathbf{p}, \boldsymbol{a})
$$

and the potential between $D_{l}^{*}$ and $\bar{D}_{l}^{*}$ up to the second order of the $p / M_{H}$ expansion

$$
\begin{aligned}
V^{2+}(\mathbf{p}, \boldsymbol{a})= & -F_{1}\left(\boldsymbol{a}^{2}\right) \frac{g_{\sigma}^{2}}{a^{2}+m_{\sigma}^{2}} F_{1}\left(\boldsymbol{a}^{2}\right) \\
& -F_{2}\left(\boldsymbol{a}^{2}\right)\left(\frac{g_{\rho}^{2}}{a^{2}+m_{\rho^{0}}^{2}}+\frac{g_{1}^{2}}{a^{2}+m_{\omega}^{2}}+\frac{g_{8}^{2}}{a^{2}+m_{\phi}^{2}}\right) \\
& \times F_{2}\left(\boldsymbol{a}^{2}\right)\left[1+\frac{4 \mathbf{p}^{2}+\boldsymbol{a}^{2}}{4 E_{1} E_{2}}\right],
\end{aligned}
$$

where $a$ is the momentum of the exchanged meson; $b^{2}(M)=\left[M^{2}-\left(M_{1}+M_{2}\right)^{2}\right]\left[M^{2}-\left(M_{1}-M_{2}\right)^{2}\right] /\left(4 M^{2}\right)$; $\mu_{R}=E_{1} E_{2} /\left(E_{1}+E_{2}\right)=\left[M^{4}-\left(M_{1}^{2}-M_{2}^{2}\right)^{2}\right] /\left(4 M^{3}\right) ; E_{1}=$ $\left(M^{2}-M_{2}^{2}+M_{1}^{2}\right) /(2 M) ; \quad E_{2}=\left(M^{2}-M_{1}^{2}+M_{2}^{2}\right) /(2 M) ;$ the meson-quark coupling constants $g_{\sigma}, g_{\rho}, g_{1}$, and $g_{8}$ are obtained within the QCD sum rules approach; and $F_{1}\left(\boldsymbol{a}^{2}\right)$ and $F_{2}\left(\boldsymbol{a}^{2}\right)$ are the form factors of heavy meson $D_{l}^{*}$ describing the heavy meson structure. These two form factors correspond to one light scalar and vector meson exchanges, respectively, which have been given in our previous work [17]. The values of the meson-quark coupling constants have been given in Refs. [7,17]. Numerically solving the equation (53), we can obtain the masses and wave functions of pure molecule states $D^{* 0} \bar{D}^{* 0}$ and $D^{*+} D^{*-}$ with $J^{P}=2^{+}$.
Using Eqs. (52) and (53), we can obtain $\mathcal{G}_{5}\left(P^{D \bar{D}} \cdot p, p^{2}\right)$ from the wave function $\Psi^{2^{+}}(\mathbf{p})$.

Similarly, considering the cross-terms between two pure molecule states $D^{* 0} \bar{D}^{* 0}$ and $D^{*+} D^{*-}$ and using the coupled-channel approach, we can obtain the mass of the $X(3915)$ state with $J^{P}=2^{+}$. The component wave function $\chi_{\lambda \tau}^{j}(P, p)$ can be obtained:

$$
\chi_{\lambda \tau}^{2^{+}}(P, p)=\frac{1}{\mathcal{N}^{2+}} \eta_{\lambda \tau}^{\varsigma} \mathcal{G}_{5}\left(P \cdot p, p^{2}\right)
$$

The reduced normalization condition for $\chi_{\lambda \tau}^{2^{+}}(P, p)$ becomes

$$
\begin{aligned}
2 P_{0}= & \frac{1}{5} \sum_{\varsigma=1}^{5} \frac{-i}{(2 \pi)^{4}} \int d^{4} p \bar{\chi}_{\lambda \tau}(P, p) \\
& \times \frac{\partial}{\partial P_{0}}\left[\Delta_{F \lambda \lambda^{\prime}}(P / 2+p)^{-1} \Delta_{F \tau \tau^{\prime}}(P / 2-p)^{-1}\right] \chi_{\lambda^{\prime} \tau^{\prime}}(P, p),
\end{aligned}
$$

where the factor $1 / 5$ appears because of the sum of five transverse directions.

Substituting Eq. (55) into (36), we obtain the GBS wave function for four-quark state $\chi_{D_{l}^{*} \bar{D}_{l}^{*}}^{2}\left(P, p, k, k^{\prime}\right)$ with $J^{P}=2^{+}$. Then, the lowest-order transition matrix element for the radiative two-photon decay of $X(3915)$ expressed as Eq. (35) becomes

$$
\begin{aligned}
\left\langle\gamma \gamma\left|S^{(2)}\right| X(3915)\right\rangle= & \frac{2}{\sqrt{2}} \frac{1}{(2 \pi)^{3 / 2}} \frac{1}{\sqrt{2\left|\mathbf{Q}^{\prime}\right|}} \frac{1}{\sqrt{2|\mathbf{Q}|}} \frac{1}{\sqrt{2 E(P)}} \frac{1}{(2 \pi)^{3}} \varepsilon_{\mu}^{\ell^{\prime *}}\left(Q^{\prime}\right) \varepsilon_{\nu}^{\Theta^{*}}(Q) \\
& \times(2 \pi)^{4} \delta^{(4)}\left(P-Q-Q^{\prime}\right)\left(\frac{4 e^{2}}{9} \mathcal{M}_{\nu \mu \lambda \tau}^{D^{* 0} \bar{D}^{* 0}}-\frac{2 e^{2}}{9} \mathcal{M}_{\nu \mu \lambda \tau}^{D^{*+} D^{*-}}\right) \eta_{\lambda \tau}^{\varsigma},
\end{aligned}
$$

where

$$
\begin{aligned}
\mathcal{M}_{\nu \mu \lambda \tau}^{D_{l}^{*} \bar{D}_{l}^{*}}= & \int d^{4} k d^{4} p \frac{1}{p_{1}^{2}+m_{c}^{2}} \frac{1}{\mathcal{N}^{D_{l}^{*}}} \frac{\varphi_{D_{l}^{*}}\left(k^{2}\right)}{k^{2}+m_{l}^{2}} \frac{1}{\mathcal{N}^{\bar{D}_{l}^{*}}} \frac{\varphi_{\bar{D}_{l}^{*}}\left(k^{\prime 2}\right)}{k^{\prime 2}+m_{l}^{2}} \frac{1}{p_{2}^{2}+m_{c}^{2}} \frac{1}{\mathcal{N}^{2^{+}}} \mathcal{G}_{5}\left(P \cdot p, p^{2}\right) \\
& \times \operatorname{Tr}\left\{\gamma_{\nu}\left[\gamma \cdot p_{1}+i m_{c}\right]\left(\gamma_{\lambda}+p_{1 \lambda}^{\prime} \frac{\gamma \cdot p_{1}^{\prime}}{M_{D_{l}^{*}}^{2}}\right)\left[\gamma \cdot(-k)+i m_{l}\right] \gamma_{\mu}\left[\gamma \cdot\left(-k^{\prime}\right)+i m_{l}\right]\right. \\
& \left.\times\left(\gamma_{\tau}+p_{2 \tau}^{\prime} \frac{\gamma \cdot p_{2}^{\prime}}{M_{\bar{D}_{l}^{*}}^{2}}\right)\left[\gamma \cdot p_{2}+i m_{c}\right]\right\} .
\end{aligned}
$$

Applying the same method mentioned in Sec. IV. A, we can calculate the tensor $\mathcal{M}_{\nu \mu \lambda \tau}^{D_{l}^{*} \bar{D}_{l}^{*}}$ (see details in Appendix B). Finally, the radiative two-photon decay width of $X(3915)$ with $J^{P}=2^{+}$should be

$$
\begin{aligned}
\Gamma^{2^{+}}= & \int d^{3} Q d^{3} Q^{\prime}(2 \pi)^{4} \delta^{(4)}\left(P-Q-Q^{\prime}\right) 2 \frac{1}{2\left|\mathbf{Q}^{\prime}\right|} \frac{1}{2|\mathbf{Q}|} \frac{1}{2 E(P)} \frac{1}{(2 \pi)^{6}} \\
& \times \frac{1}{5} \sum_{\varrho^{\prime}=1}^{2} \sum_{\varrho=1}^{2} \sum_{\varsigma=1}^{5}\left|\varepsilon_{\mu}^{\varrho^{\prime}}\left(Q^{\prime}\right) \varepsilon_{\nu}^{\varrho}(Q)\left(\frac{4 e^{2}}{9} \mathcal{M}_{\nu \mu \lambda \tau}^{D^{* 0} \bar{D}^{* 0}}-\frac{2 e^{2}}{9} \mathcal{M}_{\nu \mu \lambda \tau}^{D^{*+} D^{*-}}\right) \eta_{\lambda \tau}^{\varsigma}\right|^{2} .
\end{aligned}
$$


To calculate the normalization $\mathcal{N}^{2+}$ and the decay width $\Gamma^{2^{+}}$, we use the spin projector given in Ref. [26],

$$
\sum_{\varsigma=1}^{5} \eta_{\lambda \tau}^{\varsigma} \eta_{\lambda^{\prime} \tau^{\prime}}^{\kappa^{\prime}}=\frac{1}{2}\left(\Delta_{\lambda \lambda^{\prime}} \Delta_{\tau \tau^{\prime}}+\Delta_{\lambda \tau^{\prime}} \Delta_{\tau \lambda^{\prime}}\right)-\frac{1}{3} \Delta_{\lambda \tau} \Delta_{\lambda^{\prime} \tau^{\prime}},
$$

where $\Delta_{\lambda \tau}=\delta_{\lambda \tau}+P_{\lambda} P_{\tau} / M^{2}$.

\section{NUMERICAL RESULT}

Since the isospin conservation, we have the constituent quark masses $m_{u}=m_{d}=0.33 \mathrm{GeV}$, and $m_{c}=1.55 \mathrm{GeV}$ [6] and the meson masses $M_{D^{* 0}}=M_{D^{*+}}=2.007 \mathrm{GeV}$ [16]. Taking $\omega_{D^{*}}=1.50 \mathrm{GeV}$ as in Ref. [21] and using the approach given in Ref. [7], we recalculate the masses and wave functions of pure molecule states $D^{* 0} \bar{D}^{* 0}$ and $D^{*+} D^{*-}$ with $J^{P}=0^{+}$and $2^{+}$. Considering the cross-terms between these two pure molecule states $D^{* 0} \bar{D}^{* 0}$ and $D^{*+} D^{*-}$ and using the coupled-channel approach, we

TABLE I. Mass and widths of mixed state of two S-wave molecule states $D^{* 0} \bar{D}^{* 0}$ and $D^{*+} D^{*-}$ with $0^{+}$. We vary constituent quark masses to estimate the uncertainties.

\begin{tabular}{lccc}
\hline \hline Quantity & $m_{u, d}=0.32 \mathrm{GeV}$ & $m_{u, d}=0.33 \mathrm{GeV}$ & $m_{u, d}=0.34 \mathrm{GeV}$ \\
\hline $\begin{array}{l}\text { Mass }(\mathrm{GeV}) \\
\Gamma(X \rightarrow J / \psi \omega)\end{array}$ & 3.920 & 3.926 & 3.933 \\
$(\mathrm{MeV})$ & 44 & 49 & 64 \\
$\begin{array}{c}\Gamma \rightarrow \gamma \gamma) \\
(\mathrm{eV})\end{array}$ & 28 & 34 & 26 \\
\hline \hline
\end{tabular}

TABLE II. Mass and widths of mixed state of two S-wave molecule states $D^{* 0} \bar{D}^{* 0}$ and $D^{*+} D^{*-}$ with $2^{+}$. We vary constituent quark masses to estimate the uncertainties.

\begin{tabular}{lccc}
\hline \hline Quantity & $m_{u, d}=0.32 \mathrm{GeV}$ & $m_{u, d}=0.33 \mathrm{GeV}$ & $m_{u, d}=0.34 \mathrm{GeV}$ \\
\hline $\begin{array}{l}\text { Mass }(\mathrm{GeV}) \\
\Gamma(X \rightarrow J / \psi \omega)\end{array}$ & 3.934 & 3.941 & 3.949 \\
$(\mathrm{MeV})$ & 5 & 6 & 8 \\
$\begin{array}{l}\Gamma \rightarrow \gamma \gamma) \\
(\mathrm{eV})\end{array}$ & 10 & 12 & 9 \\
\hline \hline
\end{tabular}

obtain the masses of the mixed states with $J^{P}=0^{+}$and $2^{+}$. Then, by doing the numerical calculation, we obtain the radiative two-photon decay widths $\Gamma(X(3915) \rightarrow \gamma \gamma)$ for $J^{P}=0^{+}$and $2^{+}$. In Ref. [9], we introduced a method to evaluate the decay width of the molecular state into a heavy meson plus a light meson. Applying this method given in Ref. [9], we recalculate the strong $X(3915) \rightarrow J / \psi \omega$ decay widths for $J^{P}=0^{+}$and $2^{+}$. Our numerical results are presented in Tables I and II.

In our approach, we require the meson-quark coupling constants and the parameters $\omega_{H}$ in BS amplitudes of heavy vector mesons to calculate the mass and decay widths of $X(3915)$. These parameters are determined by providing fits to observables, which cannot be adjusted freely. Our approach also involves the constituent quark masses $m_{u}$, $m_{d}$, and $m_{c}$. According to the spontaneous breaking of chiral symmetry, the light quarks $(u, d$, and $s)$ obtain their constituent masses because the vacuum condensate is not equal to zero, and the heavy quark masses $(c, b$, and $t)$ are irrelevant to the vacuum condensate. The constituent masses of light quarks have not been exactly determined, while the large uncertainty should not exist for heavy quarks. Normally, the value slightly greater than a third of the nucleon mass is employed as the constituent mass of the light quark. Taking different values for constituent masses of light quarks $m_{u, d}$, we do the numerical calculation again and obtain the uncertainties given in Tables I and II. These numerical results imply that in our approach the calculated masses and decay widths depend on the values of constituent quark masses, but not sensitively.

In experiments, the observation of narrow state $X(3915)$ has been reported by a series of works collected in Table III. Comparing our numerical results with experimental data, we find that the calculated masses of mixed states consisting of two components $D^{* 0} \bar{D}^{* 0}$ and $D^{*+} D^{*-}$ with $J^{P}=0^{+}$and $2^{+}$are consistent with experimental data, as mentioned in Ref. [11]. However, for the strong decay and radiative two-photon decay of a mixed state consisting of $D^{* 0} \bar{D}^{* 0}$ and $D^{*+} D^{*-}$, we find that the numerical results for $J^{P}=2^{+}$are more consistent with the latest experimental data $[10,11]$ than the ones for $J^{P}=0^{+}$. In particular, the calculated radiative two-photon decay width of the mixed state with $J^{P}=2^{+}$is in good agreement

TABLE III. Experimental data on the mass and decay widths of $X(3915)$, given in chronological order. $\mathcal{B}$ is the branching fraction.

\begin{tabular}{llccc}
\hline \hline Year & Experiment & Mass $(\mathrm{MeV})$ & $\Gamma(X \rightarrow J / \psi \omega)(\mathrm{MeV})$ & $\Gamma(X \rightarrow \gamma \gamma) \times \mathcal{B}(X \rightarrow J / \psi \omega)(\mathrm{eV})$ \\
\hline 2005 & Belle [12] & $3943 \pm 11 \pm 13$ & $87 \pm 22 \pm 26$ & \\
2008 & $B A B A R[13]$ & $3914.6_{-3.4}^{+3.8} \pm 2.0$ & $34_{-8}^{+12} \pm 5$ & $61 \pm 17 \pm 8$ for $J^{P}=0^{+}$ \\
2010 & Belle [10] & $3915 \pm 3 \pm 2$ & $17 \pm 10 \pm 3$ & $18 \pm 5 \pm 2$ for $J^{P}=2^{+}$ \\
2012 & & & $13 \pm 6 \pm 3$ & $52 \pm 10 \pm 3$ for $J^{P}=0^{+}$ \\
& BABAR [11] & $3919.4 \pm 2.2 \pm 1.6$ & & $10.5 \pm 1.9 \pm 0.6$ for $J^{P}=2^{+}$ \\
\hline \hline
\end{tabular}


with the latest experimental data, which provide further verification for the discrimination of the $X(3915)$ quantum numbers.

We summarize the numerical results as follows: the strong decay width is $\Gamma(X(3915) \rightarrow J / \psi \omega)=6 \mathrm{MeV}$, the radiative two-photon decay width is $\Gamma(X(3915) \rightarrow \gamma \gamma)=12 \mathrm{eV}$, and the quantum numbers of the $X(3915)$ state are $J^{P}=2^{+}$. To investigate the radiative decay of a mixed state consisting of two components, we consider the photon interaction with quarks, which can be described by the exact interaction Lagrangian. Therefore, this work provides a further verification for the molecular hypothesis of $X(3915)$ and predicts the exact value of radiative two-photon decay width $\Gamma(X(3915) \rightarrow \gamma \gamma)$.

Up to now, a systematical and accurate theoretical approach from QCD to investigate the molecular state composed of two heavy vector mesons has been established. Applying the general form of the Bethe-Salpeter wave functions for the bound states composed of two vector fields, we calculated the mass of molecular state and obtained its BS wave function [7]. Then, using the general form of the GBS wave functions for four-quark states describing the meson-meson molecular structure, we calculated the decay width of the molecular state into a heavy meson plus a light meson [9]. In this work, we investigate the radiative two-photon decay of the molecular state. From the effective theory at low-energy QCD, we investigate the molecular state consisting of two vector mesons, which is the main difference between our approach and these other works about hadronic molecule states.

\section{CONCLUSION}

The general form of the GBS wave functions for fourquark states describing the molecular structure is applied to investigate the radiative two-photon decay of a molecular state composed of two vector mesons, and the general formulas for the two-photon decay widths of molecular states is obtained. Then, assuming that the exotic state $X(3915)$ is a mixed state of two components $D^{* 0} \bar{D}^{* 0}$ and $D^{*+} D^{*-}$, we carefully consider the internal structure of the vector mesons in the molecular state and numerically calculate the decay widths $\Gamma(X(3915) \rightarrow \gamma \gamma)$ with $J^{P}=0^{+}$ and $2^{+}$. Comparing our numerical results with experimental data, we deduce that the quantum numbers of the $X(3915)$ state are $J^{P}=2^{+}$. From the effective theory at low-energy QCD and the Weinberg-Salam model, we have systematically investigated the strong and radiative decay modes of a molecular state composed of two heavy vector mesons. In the future, to contain more inspiration of QCD, we will introduce the nonperturbative contribution from vacuum condensates into the GBS wave function for the four-quark state and the irreducible part of the Green's function.

\section{ACKNOWLEDGMENTS}

This work was supported by the National Natural Science Foundation of China under Grants No. 11705104 and No. 11801323; Shandong Provincial Natural Science Foundation, China, under Grants No. ZR2016AQ19 and No. ZR2016AM31; a project of Shandong Province Higher Education Science and Technology program under Grants No. J17KB130 and No. J18KA227; and SDUST Research Fund under Grant No. 2018TDJH101.

\section{APPENDIX A: THE TENSOR STRUCTURES IN THE GENERAL FORM OF THE BS WAVE FUNCTIONS}

The tensor structures in Eqs. (12)-(15) are given below [7]:

$$
\begin{aligned}
T_{\lambda \tau}^{1}= & \left(\eta_{1} \eta_{2} P^{2}-\eta_{1} P \cdot p+\eta_{2} P \cdot p-p^{2}\right) g_{\lambda \tau}-\left(\eta_{1} \eta_{2} P_{\lambda} P_{\tau}+\eta_{2} P_{\lambda} p_{\tau}-\eta_{1} p_{\lambda} P_{\tau}-p_{\lambda} p_{\tau}\right), \\
T_{\lambda \tau}^{2}= & \left(\eta_{1}^{2} P^{2}+2 \eta_{1} P \cdot p+p^{2}\right)\left(\eta_{2}^{2} P^{2}-2 \eta_{2} P \cdot p+p^{2}\right) g_{\lambda \tau} \\
& +\left(\eta_{1} \eta_{2} P^{2}-\eta_{1} P \cdot p+\eta_{2} P \cdot p-p^{2}\right)\left(\eta_{1} \eta_{2} P_{\lambda} P_{\tau}-\eta_{1} P_{\lambda} p_{\tau}+\eta_{2} p_{\lambda} P_{\tau}-p_{\lambda} p_{\tau}\right) \\
& -\left(\eta_{2}^{2} P^{2}-2 \eta_{2} P \cdot p+p^{2}\right)\left(\eta_{1}^{2} P_{\lambda} P_{\tau}+\eta_{1} P_{\lambda} p_{\tau}+\eta_{1} p_{\lambda} P_{\tau}+p_{\lambda} p_{\tau}\right) \\
& -\left(\eta_{1}^{2} P^{2}+2 \eta_{1} P \cdot p+p^{2}\right)\left(\eta_{2}^{2} P_{\lambda} P_{\tau}-\eta_{2} P_{\lambda} p_{\tau}-\eta_{2} p_{\lambda} P_{\tau}+p_{\lambda} p_{\tau}\right), \\
T_{\mu_{1} \cdots \mu_{j} \lambda \tau}^{3}= & \frac{1}{j !} p_{\left\{\mu_{2}\right.} \cdots p_{\mu_{j}} g_{\left.\mu_{1}\right\} \lambda}\left(\eta_{1}^{2} P^{2}+2 \eta_{1} P \cdot p+p^{2}\right)\left[\left(\eta_{2}^{2} P^{2}-2 \eta_{2} P \cdot p+p^{2}\right)\left(\eta_{1} P+p\right)_{\tau}\right. \\
& \left.-\left(\eta_{1} \eta_{2} P^{2}-\eta_{1} P \cdot p+\eta_{2} P \cdot p-p^{2}\right)\left(\eta_{2} P-p\right)_{\tau}\right] \\
& -p_{\mu_{1}} \cdots p_{\mu_{j}}\left[\left(\eta_{2}^{2} P^{2}-2 \eta_{2} P \cdot p+p^{2}\right)\left(\eta_{1}^{2} P_{\lambda} P_{\tau}+\eta_{1} P_{\lambda} p_{\tau}+\eta_{1} p_{\lambda} P_{\tau}+p_{\lambda} p_{\tau}\right)\right. \\
& \left.-\left(\eta_{1} \eta_{2} P^{2}-\eta_{1} P \cdot p+\eta_{2} P \cdot p-p^{2}\right)\left(\eta_{1} \eta_{2} P_{\lambda} P_{\tau}-\eta_{1} P_{\lambda} p_{\tau}+\eta_{2} p_{\lambda} P_{\tau}-p_{\lambda} p_{\tau}\right)\right]
\end{aligned}
$$




$$
\begin{aligned}
& T_{\mu_{1} \cdots \mu_{j} \lambda \tau}^{4}=\frac{1}{j !} p_{\left\{\mu_{2}\right.} \cdots p_{\mu_{j}} g_{\left.\mu_{1}\right\} \tau}\left(\eta_{2}^{2} P^{2}-2 \eta_{2} P \cdot p+p^{2}\right)\left[\left(\eta_{1} \eta_{2} P^{2}-\eta_{1} P \cdot p\right.\right. \\
& \left.\left.+\eta_{2} P \cdot p-p^{2}\right)\left(\eta_{1} P+p\right)_{\lambda}-\left(\eta_{1}^{2} P^{2}+2 \eta_{1} P \cdot p+p^{2}\right)\left(\eta_{2} P-p\right)_{\lambda}\right] \\
& -p_{\mu_{1}} \cdots p_{\mu_{j}}\left[\left(\eta_{1}^{2} P^{2}+2 \eta_{1} P \cdot p+p^{2}\right)\left(\eta_{2}^{2} P_{\lambda} P_{\tau}-\eta_{2} P_{\lambda} p_{\tau}-\eta_{2} p_{\lambda} P_{\tau}+p_{\lambda} p_{\tau}\right)\right. \\
& \left.-\left(\eta_{1} \eta_{2} P^{2}-\eta_{1} P \cdot p+\eta_{2} P \cdot p-p^{2}\right)\left(\eta_{1} \eta_{2} P_{\lambda} P_{\tau}-\eta_{1} P_{\lambda} p_{\tau}+\eta_{2} p_{\lambda} P_{\tau}-p_{\lambda} p_{\tau}\right)\right], \\
& T_{\mu_{1} \cdots \mu_{j} \lambda \tau}^{5}=\left(\eta_{1}^{2} P^{2}+2 \eta_{1} P \cdot p+p^{2}\right)\left(\eta_{2}^{2} P^{2}-2 \eta_{2} P \cdot p+p^{2}\right) p_{\mu_{3}} \cdots p_{\mu_{j}} g_{\mu_{1} \lambda} g_{\mu_{2} \tau} \\
& -\frac{1}{j !} p_{\left\{\mu_{2}\right.} \cdots p_{\mu_{j}} g_{\left.\mu_{1}\right\} \tau}\left(\eta_{2}^{2} P^{2}-2 \eta_{2} P \cdot p+p^{2}\right)\left(\eta_{1} P+p\right)_{\lambda}+\frac{1}{j !} p_{\left\{\mu_{2}\right.} \cdots p_{\mu_{j}} g_{\left.\mu_{1}\right\} \lambda}\left(\eta_{1}^{2} P^{2}+2 \eta_{1} P \cdot p+p^{2}\right)\left(\eta_{2} P-p\right)_{\tau} \\
& -p_{\mu_{1}} \cdots p_{\mu_{j}}\left(\eta_{1} \eta_{2} P_{\lambda} P_{\tau}-\eta_{1} P_{\lambda} p_{\tau}+\eta_{2} p_{\lambda} P_{\tau}-p_{\lambda} p_{\tau}\right), \\
& T_{\mu_{1} \cdots \mu_{j} \lambda \tau}^{6}=\left(\eta_{2} P \cdot p-\eta_{1} P \cdot p-2 p^{2}\right) p_{\left\{\mu_{2}\right.} \cdots p_{\mu_{j}} \epsilon_{\left.\mu_{1}\right\} \lambda \tau \xi} p_{\xi}+\left(2 \eta_{1} \eta_{2} P \cdot p+\eta_{2} p^{2}-\eta_{1} p^{2}\right) p_{\left\{\mu_{2}\right.} \cdots p_{\mu_{j}} \epsilon_{\left.\mu_{1}\right\} \lambda \tau \xi} P_{\xi} \\
& +p_{\left\{\mu_{2}\right.} \cdots p_{\mu_{j}} \epsilon_{\left.\mu_{1}\right\} \lambda \xi \zeta} p_{\xi} P_{\zeta} p_{\tau}+p_{\left\{\mu_{2}\right.} \cdots p_{\mu_{j}} \epsilon_{\left.\mu_{1}\right\} \tau \xi \zeta} p_{\xi} P_{\zeta} p_{\lambda}, \\
& T_{\mu_{1} \cdots \mu_{j} \lambda \tau}^{7}=(P \cdot p) p_{\left\{\mu_{2}\right.} \cdots p_{\mu_{j}} \epsilon_{\left.\mu_{1}\right\} \lambda \tau \xi} p_{\xi}-p^{2} p_{\left\{\mu_{2}\right.} \cdots p_{\mu_{j}} \epsilon_{\left.\mu_{1}\right\} \lambda \tau \xi} P_{\xi}+p_{\left\{\mu_{2}\right.} \cdots p_{\mu_{j}} \epsilon_{\left.\mu_{1}\right\} \lambda \xi \zeta} p_{\xi} P_{\zeta} p_{\tau}-p_{\left\{\mu_{2}\right.} \cdots p_{\mu_{j}} \epsilon_{\left.\mu_{1}\right\} \tau \xi \zeta} p_{\xi} P_{\zeta} p_{\lambda}, \\
& T_{\mu_{1} \cdots \mu_{j} \lambda \tau}^{8}=\left(\eta_{2} P^{2}-\eta_{1} P^{2}-2 P \cdot p\right) p_{\left\{\mu_{2}\right.} \cdots p_{\mu_{j}} \epsilon_{\left.\mu_{1}\right\} \lambda \tau \xi} p_{\xi}+\left(2 \eta_{1} \eta_{2} P^{2}+\eta_{2} P \cdot p-\eta_{1} P \cdot p\right) p_{\left\{\mu_{2}\right.} \cdots p_{\mu_{j}} \epsilon_{\left.\mu_{1}\right\} \lambda \tau \xi} P_{\xi} \\
& +p_{\left\{\mu_{2}\right.} \cdots p_{\mu_{j}} \epsilon_{\left.\mu_{1}\right\} \lambda \xi \zeta} p_{\xi} P_{\zeta} P_{\tau}+p_{\left\{\mu_{2}\right.} \cdots p_{\mu_{j}} \epsilon_{\left.\mu_{1}\right\} \tau \xi \zeta} p_{\xi} P_{\zeta} P_{\lambda} \\
& T_{\mu_{1} \cdots \mu_{j} \lambda \tau}^{9}=P^{2} p_{\left\{\mu_{2}\right.} \cdots p_{\mu_{j}} \epsilon_{\left.\mu_{1}\right\} \lambda \tau \xi} p_{\xi}-(P \cdot p) p_{\left\{\mu_{2}\right.} \cdots p_{\mu_{j}} \epsilon_{\left.\mu_{1}\right\} \lambda \tau \xi} P_{\xi}+p_{\left\{\mu_{2}\right.} \cdots p_{\mu_{j}} \epsilon_{\left.\mu_{1}\right\} \lambda \xi \zeta} p_{\xi} P_{\zeta} P_{\tau}-p_{\left\{\mu_{2}\right.} \cdots p_{\mu_{j}} \epsilon_{\left.\mu_{1}\right\} \tau \xi \zeta} p_{\xi} P_{\zeta} P_{\lambda} \text {. }
\end{aligned}
$$

\section{APPENDIX B: CALCULATION OF TENSOR $\mathcal{M}_{\nu \mu \lambda \tau}^{D_{i}^{*} \bar{D}_{i}^{*}}$ IN THE DECAY WIDTH OF $X(3915)$ WITH $J^{P}=2^{+}$}

Because the tensor $\mathcal{M}_{\nu \mu \lambda \tau}^{D_{l}^{*} \bar{D}_{l}^{*}}$ in Eq. (58) also depends on $P$ and $Q$, it can be expressed as

$$
\begin{aligned}
\mathcal{M}_{\nu \mu \lambda \tau}^{D_{l}^{*} \bar{D}_{l}^{*}}= & g_{\nu \mu} g_{\lambda \tau} R_{1}+g_{\nu \lambda} g_{\mu \tau} R_{2}+g_{\nu \tau} g_{\mu \lambda} R_{3}+g_{\nu \mu} P_{\lambda} P_{\tau} R_{4}+g_{\nu \mu} P_{\lambda} Q_{\tau} R_{5}+g_{\nu \mu} Q_{\lambda} P_{\tau} R_{6}+g_{\nu \mu} Q_{\lambda} Q_{\tau} R_{7} \\
& +g_{\nu \lambda} P_{\mu} P_{\tau} R_{8}+g_{\nu \lambda} P_{\mu} Q_{\tau} R_{9}+g_{\nu \lambda} Q_{\mu} P_{\tau} R_{10}+g_{\nu \lambda} Q_{\mu} Q_{\tau} R_{11}+g_{\nu \tau} P_{\mu} P_{\lambda} R_{12}+g_{\nu \tau} P_{\mu} Q_{\lambda} R_{13}+g_{\nu \tau} Q_{\mu} P_{\lambda} R_{14} \\
& +g_{\nu \tau} Q_{\mu} Q_{\lambda} R_{15}+g_{\mu \lambda} P_{\nu} P_{\tau} R_{16}+g_{\mu \lambda} P_{\nu} Q_{\tau} R_{17}+g_{\mu \lambda} Q_{\nu} P_{\tau} R_{18}+g_{\mu \lambda} Q_{\nu} Q_{\tau} R_{19}+g_{\mu \tau} P_{\nu} P_{\lambda} R_{20}+g_{\mu \tau} P_{\nu} Q_{\lambda} R_{21} \\
& +g_{\mu \tau} Q_{\nu} P_{\lambda} R_{22}+g_{\mu \tau} Q_{\nu} Q_{\lambda} R_{23}+g_{\lambda \tau} P_{\nu} P_{\mu} R_{24}+g_{\lambda \tau} P_{\nu} Q_{\mu} R_{25}+g_{\lambda \tau} Q_{\nu} P_{\mu} R_{26}+g_{\lambda \tau} Q_{\nu} Q_{\mu} R_{27} \\
& +P_{\nu} Q_{\mu} Q_{\lambda} Q_{\tau} R_{28}+P_{\mu} Q_{\nu} Q_{\lambda} Q_{\tau} R_{29}+P_{\lambda} Q_{\nu} Q_{\mu} Q_{\tau} R_{30}+P_{\tau} Q_{\nu} Q_{\mu} Q_{\lambda} R_{31}+P_{\nu} P_{\mu} Q_{\lambda} Q_{\tau} R_{32}+P_{\nu} P_{\lambda} Q_{\mu} Q_{\tau} R_{33} \\
& +P_{\nu} P_{\tau} Q_{\mu} Q_{\lambda} R_{34}+P_{\mu} P_{\lambda} Q_{\nu} Q_{\tau} R_{35}+P_{\mu} P_{\tau} Q_{\nu} Q_{\lambda} R_{36}+P_{\lambda} P_{\tau} Q_{\nu} Q_{\mu} R_{37}+P_{\mu} P_{\lambda} P_{\tau} Q_{\nu} R_{38}+P_{\nu} P_{\lambda} P_{\tau} Q_{\mu} R_{39} \\
& +P_{\nu} P_{\mu} P_{\tau} Q_{\lambda} R_{40}+P_{\nu} P_{\mu} P_{\lambda} Q_{\tau} R_{41}+P_{\nu} P_{\mu} P_{\lambda} P_{\tau} R_{42}+Q_{\nu} Q_{\mu} Q_{\lambda} Q_{\tau} R_{43},
\end{aligned}
$$

where $R_{i}(P, Q)(i=1, \ldots, 43)$ are the scalar functions. There are 43 tensor structures in the above expression. Then, Eq. (B1) is multiplied by these 43 tensor structures respectively; and a system of 43 equations is obtained. Subsequently, we numerically calculate the products of Eq. (B1) and these 43 tensor structures, respectively, and solve this system of equations. The values of $R_{i}$ can be obtained. Obviously, the expression of tensor $\mathcal{M}_{\nu \mu \lambda \tau}^{D_{l}^{*} \bar{D}_{l}^{*}}$ given in Eq. (B1) is a general formalism, which can be used to evaluate the strong decay width of $X(3915)$ with $J^{P}=2^{+}$. 
[1] E. S. Swanson, Phys. Lett. B 588, 189 (2004).

[2] N. A. Törnqvist, Phys. Lett. B 590, 209 (2004).

[3] T. Branz, T. Gutsche, and V. E. Lyubovitskij, Phys. Rev. D 80, 054019 (2009).

[4] L. Maiani, F. Piccinini, A. D. Polosa, and V. Riquer, Phys. Rev. D 71, 014028 (2005).

[5] L. Maiani, A. D. Polosa, and V. Riquer, Phys. Rev. Lett. 99, 182003 (2007).

[6] D. Ebert, R. N. Faustov, and V. O. Galkin, Phys. Lett. B 634, 214 (2006).

[7] X. Chen and X. Lü, Eur. Phys. J. C 75, 98 (2015).

[8] X. Chen, X. Lü, R. Shi, and X. Guo, Nucl. Phys. B909, 243 (2016).

[9] X. Chen and X. Lü, Phys. Rev. D 97, 114005 (2018).

[10] S. Uehara et al. (Belle Collaboration), Phys. Rev. Lett. 104, 092001 (2010).

[11] J. P. Lees et al. (BABAR Collaboration), Phys. Rev. D 86, 072002 (2012).

[12] S.-K. Choi et al. (Belle Collaboration), Phys. Rev. Lett. 94, 182002 (2005).

[13] B. Aubert et al. (BABAR Collaboration), Phys. Rev. Lett. 101, 082001 (2008).

[14] T. Aaltonen et al. (CDF Collaboration), Phys. Rev. Lett. 102, 242002 (2009).
[15] A. Esposito, A. Pilloni, and A. D. Polosa, Phys. Rep. 668, 1 (2017).

[16] M. Tanabashi et al. (Particle Data Group), Phys. Rev. D 98, 030001 (2018).

[17] X. Chen, R. Liu, R. Shi, and X. Lü, Phys. Rev. D 87, 065013 (2013).

[18] C. J. Burden, L. Qian, C. D. Roberts, P. C. Tandy, and M. J. Thomson, Phys. Rev. C 55, 2649 (1997).

[19] P. Maris, C. D. Roberts, and P. C. Tandy, Phys. Lett. B 420, 267 (1998).

[20] M. A. Ivanov, Y. L. Kalinovsky, and C. D. Roberts, Phys. Rev. D 60, 034018 (1999).

[21] M. A. Ivanov, J. G. Körner, S. G. Kovalenko, and C. D. Roberts, Phys. Rev. D 76, 034018 (2007).

[22] D. Lurié, Particles and Fields (Interscience, New York, 1968).

[23] X. Liu and S. L. Zhu, Phys. Rev. D 80, 017502 (2009).

[24] F. E. Close and E. S. Swanson, Phys. Rev. D 72, 094004 (2005).

[25] M. Neubert, Phys. Rep. 245, 259 (1994).

[26] A. M. Torres, K. P. Khemchandani, M. Nielsen, F. S. Navarra, and E. Oset, Phys. Rev. D 88, 074033 (2013). 